\title{
Binding methylarginines and methyllysines as free amino acids: a comparative study of multiple supramolecular host classes
}

\author{
Zoey Warmerdam, ${ }^{1, a}$ Bianca E. Kamba,,${ }^{1, b}$ My-Hue Le,${ }^{c}$ Thomas Schrader, ${ }^{c}$ Lyle Isaacs, ${ }^{d^{*}}$ Peter Bayer, ${ }^{b^{*}}$ \\ and Fraser Hof ${ }^{\text {a* }}$
}

Dedicated to François Diederich, enthusiastic promotor of collaboration and partnerships

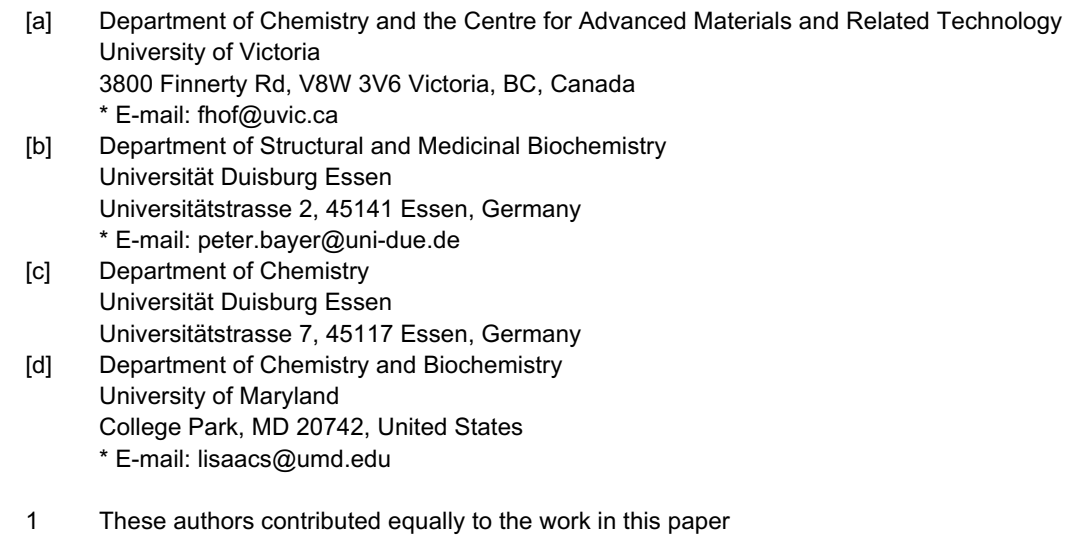

Supporting information for this article is given via a link at the end of the document.

\begin{abstract}
Methylated free amino acids are an important class of targets for host-guest chemistry that have recognition properties distinct from those of methylated peptides and proteins. We present comparative binding studies for three different host classes that are each studied with multiple methylated arginines and lysines to determine fundamental structure-function relationships. The hosts studied are all anionic and include three calixarenes, two acyclic cucurbiturils, and two cleft-like hosts. We determined the binding association constants for a panel of methylated amino acids using indicator displacement assays. The calixarene hosts show weak binding that favours the higher methylation states, with the strongest binding observed for trimethyllysine. The acyclic cucurbiturils display stronger binding to the methylated amino acids, and some unique patterns of selectivity. The cleft-like hosts follow two different trends, one shallow host following similar trends to the calixarenes, and the other more closed host binding certain less-methylated amino acids stronger than their per-methylated counterparts. Molecular modeling sheds some light on the different preferences of different hosts. The results identify hosts with selectivities that will be useful for certain biomedical applications. The overall selectivity patterns are explained by a common framework that considers the topology, depth of binding pockets, and functional group participation across all host classes.
\end{abstract}

\section{Introduction}

The selective binding of free amino acids in physiologically relevant solutions is difficult to achieve. Amino acids are small and zwitterionic, and the relatively small hydrophobic surface area combined with relatively high charge means that they are strongly solvated by water. The common presence of salts and other cosolutes creates further challenges. ${ }^{1}$ Although rare, there are dozens of diseases caused by disordered amino acid metabolism. ${ }^{2,3}$ There are also multiple methylated amino acids whose concentrations are diagnostic of different medical conditions, and some of them are seen as causative agents in different pathologies. Directly capturing and sequestering disease-related amino acids is a new approach that might be useful for diagnosis, disease monitoring, and possibly for direct therapeutics. ${ }^{4,5}$ Antibodies are known that bind amino acids but have some inherent shortcomings ${ }^{6-9}$ that could be overcome by the creation of synthetic organic binding tools for amino acids.

Supramolecular hosts are a great starting point to develop such new tools. The hosts have a concave binding pocket where the molecular recognition takes place via non-covalent interactions (e.g. electrostatics, hydrophobic effect, hydrogen bonding, van der Waals forces, $\pi-\pi$ interactions, etc.) These binding pockets come with different shapes, sizes and chemical properties that influence the host-guest binding properties. The recognition of amino acids by supramolecular hosts has recently been reviewed by Basílio et al. ${ }^{10}$ Examples of supramolecular hosts binding to free amino acids include calixarenes, pillararenes, cucurbiturils, cyclodextrins and many other macrocyclic molecules. Although our ability to use hosts to target small molecules is improving, there are still challenges when designing supramolecular hosts to bind hydrophilic small molecules in water. In neutral aqueous solution, free amino acids are zwitterionic and strongly hydrated, this results in unfavourable binding energy upon complexation with the host, as several water molecules need to be released from strong hydrogen bonds to the amino acid's charged groups. This hurdle becomes harder to overcome in a salty environment where, with an increasing ionic strength of the solution, a stronger competition arises through multiple mechanisms. ${ }^{1}$ 
We studied a small set of hosts from three different classes to get a better fundamental understanding of the structurefunction relationship for amino acid binding by multiple different kinds of hosts. Calixarenes are relative shallow but easily functionalized molecules, where the functionalization often directly lines the binding pocket. ${ }^{11}$ We chose a parent anionic calixarene, sCx4, and the functionalized analogs $\mathbf{s} \mathbf{C x} 4-\mathrm{NO}_{2}$ and sCx4-CHO to include in this study (Figure 1a-c). Cucurbituril (CB) hosts have a deeper and more rigid binding pocket, but the functional group additions to CBs often do not directly influence the binding properties as they happen on the outside of the host. Acyclic CB analogs such as $\mathbf{M} \mathbf{1}$ and $\mathbf{M} 2$ (Figure 1d,e) can be functionalized along the edge of their binding surfaces, and are increasingly being used in biomedical applications. ${ }^{12}$ Another more rigid cleft-like host family includes the 'tweezers' and 'clips' introduced by Klärner and Schrader. ${ }^{13,14}$ The "phosphate clip" PC carries planar aromatic sidewalls ideal for aromatic cations, whereas the "tweezers" CLR01 form a torus-shaped cavity that is selective for arginine and lysine (Figure 1f, g).

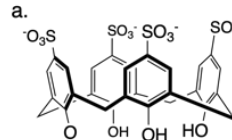

$\mathrm{sC} \times 4$

b.

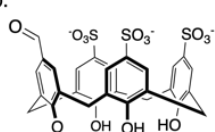

sCx4-CHO

c.

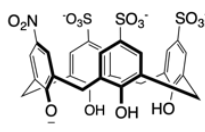

$\mathrm{sC} \times 4-\mathrm{NO}_{2}$

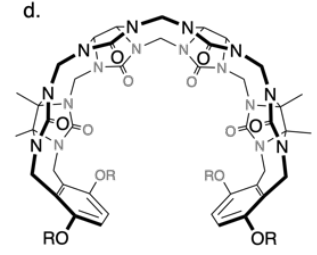

$\mathrm{M1}, \mathrm{R}=\left(\mathrm{CH}_{2}\right)_{3} \mathrm{SO}_{3}^{*}$

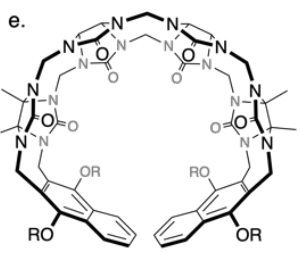

$\mathrm{M} 2, \mathrm{R}=\left(\mathrm{CH}_{2}\right)_{3} \mathrm{SO}_{3} \cdot$

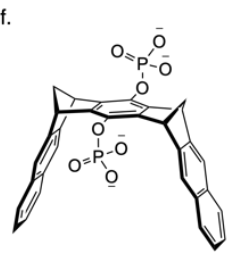

PC

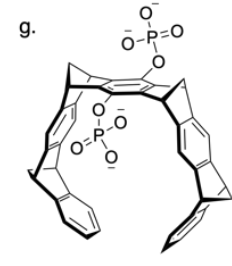

CLR01
Figure 1. Hosts studied in this report. a) sCx4. b) sCx4-CHO. c) sCx4-NO ${ }_{2}$. d) M1. e) M2. f) PC. g) CLR01.
For this study we have selected methylated amino acids as binding targets. They have very subtle structural differences among them, which make them an interesting test case for molecular recognition and selectivity. They participate in diverse biological pathways that are relevant to multiple pathological states. ${ }^{15-20}$ Our test set (Figure 2) includes arginine and lysine, and each of their physiologically relevant methylated states: (monomethyl arginine (MMA), asymmetric dimethyl arginine (ADMA), symmetric dimethyl arginine (SDMA), monomethyl lysine (MML) dimethyl lysine (DML) and trimethyl lysine (TML)). Lots of research has been focused on binding methylated lysines, ${ }^{21-24}$ while the selective binding of methylated arginine has received relatively less attention. ${ }^{25,26}$ Most prior literature has focused on binding these methylated residues in the context of proteins and peptides. This study on free amino acids is motivated by a body of literature ${ }^{15,18,27-29}$ demonstrating that some of the free methylated amino acids are metabolites that play critical biological roles in pathways that are distinct from those involving whole proteins, protein tails, and peptides with post-translationally methylated residues. As mentioned above, the existence of $\alpha$ $\mathrm{NH}_{3}{ }^{+}$and $\mathrm{CO}_{2}^{-}$groups on the free amino acids makes them challenging targets for binding in aqueous solutions. Against this backdrop, the presence of methyl groups in different numbers and arrangements is a subtle set of differences ${ }^{30}$ for hosts to distinguish.

\section{Results and Discussion}

The binding constants were determined for the complexes formed by each member of the host library with each of the guests, using indicator displacement assays (IDAs). We adapted previously reported IDAs ${ }^{31}$ for $\mathbf{s} \mathbf{C x} 4$ (using lucigenin, Figure $2 \mathrm{~b}$ ) ${ }^{32}$ and M1 and M2 (using Rhodamine 6G, Figure 2b). ${ }^{33,34}$ We established IDAs for PC and CLR01 during this work. Initial experiments using Rhodamine 6G, Proflavine, and Neutral Red revealed either non-ideal stoichiometries of binding or inadequate intensity changes upon binding. Studies using 4-ASP (Figure 2b)<smiles>CNC(=[NH2+])NCCC[C@H]([NH3+])C(=O)[O-]</smiles><smiles>NCCCNC(=O)NCCCC(N)=O</smiles><smiles>NC(=O)NCCCNC(=O)C(N)=O</smiles><smiles>CN(CCCCNN)C(=O)O</smiles><smiles>CNC(CCCCC(N)=O)C(=O)O</smiles>

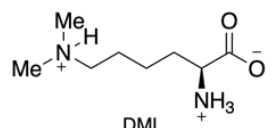<smiles>NC(CCC1CCCC1NO)C(=O)O</smiles>

b.<smiles></smiles>

Lucigenin (LCG)<smiles>CN(C)c1ccc(/C=C/c2cc[n+](C)cc2)cc1</smiles>
4-ASP<smiles>CCNc1cc2oc3cc(=[NH+]CC)c(C)cc-3c(-c3ccccc3C(=O)OCC)c2cc1C</smiles>

as the indicator gave reliable results for PC and CLR01 with the whole panel of guests. Each host-indicator dissociation constant

Figure 2. Guests and indicators studied in this report. a) Guests arginine, monomethylarginine (MMA), asymmetric dimethylarginine (ADMA), symmetric dimethylarginine (SDMA), Lysine, monomethyllysine (MML), dimethyllysine (DML), and trimethyllysine (TML), b) Indicators LCG, 4-ASP, and R6G. 
( $\left.K_{\text {ind }}\right)$ was determined by a direct titration of the host into indicator, and the host-guest $K_{d}$ values were then determined using competitive titrations of guests into a pre-formed host-indicator complex. The calixarene and the acyclic CB IDAs gave a turn-on signal, where displacement of the indicator by the guest results in an increase of fluorescence emission. The PC and CLR01 host provided a turn-off signal where displacement of 4-ASP quenches its fluorescence emission. All the titrations were optimized to work in a $10 \mathrm{mM} \mathrm{Na}_{2} \mathrm{HPO}_{4}$ buffer at $\mathrm{pH}$ 7.4. This choice of buffer rules out the ability to measure very weak binding (which would require analyte concentrations that could overwhelm the buffer), but it ensures that the trends for stronger-binding guests can be compared across different host types. The studied host-guests show a range of affinities (Table 1), which we categorize as follows for convenient presentation: strong binding $\left(K_{d}<200 \mu \mathrm{M}\right)$, medium to strong binding ( $\left.\mathrm{K}_{\mathrm{d}} 200-1500 \mu \mathrm{M}\right)$ and weak to no binding $\left(K_{d}>1500 \mu M\right)$. Exemplary primary data are presented in Figure 3 and all titrations are presented in the supportive information.
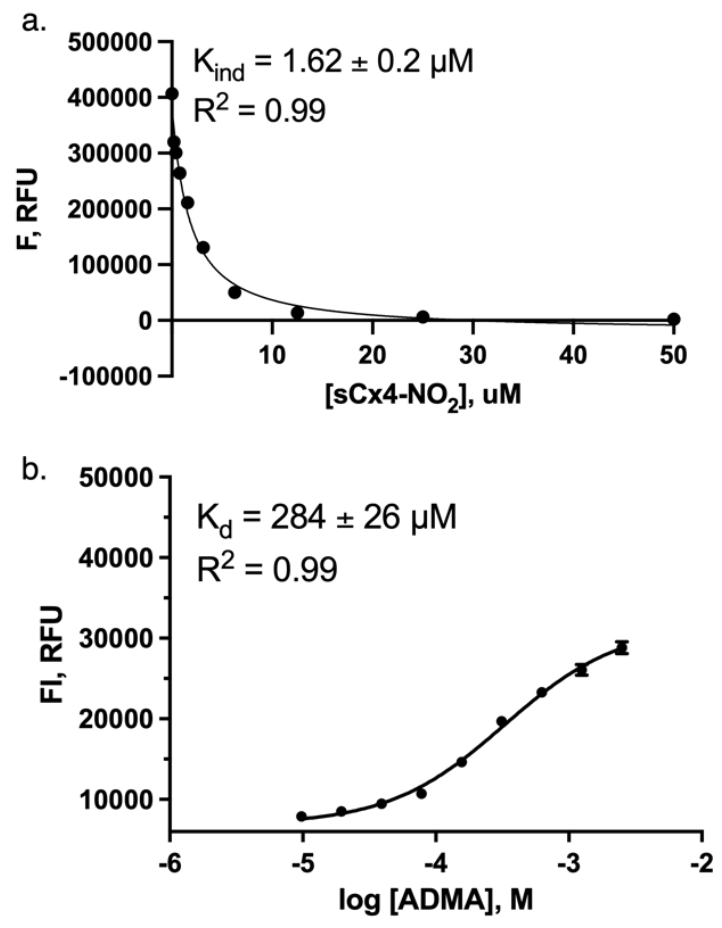

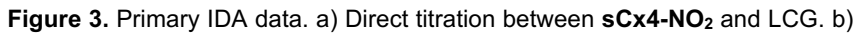
Competitive titration between $\mathbf{s} \mathbf{C x} 4-\mathrm{NO}_{2}$ and ADMA. carried out in $10 \mathrm{mM}$ $\mathrm{NaH}_{2} \mathrm{PO}_{4}$ buffer at $\mathrm{pH}$ 7.4. See the Supporting Information for titration curves, experimental details, and fitting details.
For the calixarene hosts, strong binding is only observed for a few host-guest combinations. A binding trend is observed for the calixarene-guest complexes in which higher methylation states generally result in stronger binding. Most notably, trimethyllysine (TML) is the guest that shows strongest binding with each of the sulfonatocalixarenes. This trend is less straightforward for the arginine guests although the lower methylation states show no binding within the limits of this assay condition. Comparing the symmetric and asymmetric isomers of dimethylarginine, ADMA displays a $\sim 2$-fold stronger binding. Interestingly, $\mathbf{s} \mathbf{C x} 4-\mathrm{NO}_{2}$ selectively binds ADMA over the other arginine guests, for which no binding is observed. Overall, the $\mathrm{sCx}$-hosts bind the free amino acids weakly compared to physiological concentration ranges for these amino acids, which are generally low- or sub- $\mu \mathrm{M}$.

Strong binding of the amino acids is consistently observed for M1 and M2. M1 binds all three methylated arginines, while ignoring unmethylated arginine. M1 is slightly selective for ADMA $\left(\mathrm{K}_{d}=10 \mu \mathrm{M}\right)$ over MMA $\left(\mathrm{K}_{d}=45 \mu \mathrm{M}\right)$ and SDMA $\left(\mathrm{K}_{d}=35 \mu \mathrm{M}\right)$. It has a similar selectivity for the methylated lysines showing no binding to lysine and strong binding for the methylated lysines, being selective for TML $\left(K_{d}=15 \mu \mathrm{M}\right)$ over DML $\left(K_{d}=70 \mu \mathrm{M}\right)$ and MML $\left(K_{d}=160 \mu M\right)$. M2 displays a sharp drop off between strongly binding highly methylated guests (ADMA, SDMA, DML, $\mathrm{TML}$ ) and the weak binding of lower methylation states. $\mathrm{CB}[\mathrm{n}]-$ type receptors are known to prefer quaternary over primary ammoniums. ${ }^{35}$ Among the stronger binding guests, M2 has a $>3-$ fold selectivity for SDMA $\left(K_{d}=40 \mu \mathrm{M}\right)$ over ADMA $\left(K_{d}=130 \mu M\right)$, and $a>10$-fold selectivity for TML $\left(K_{d}=30 \mu M\right)$ over DML $\left(K_{d}=\right.$ $340 \mu \mathrm{M})$.

Despite their chemical similarity, PC and CLR01 have very different behaviors. PC has relatively weak binding across the set of guests, but some trends can be observed. The higher the methylation state of the arginine guests the stronger the binding gets. When looking at the lysine guests the binding is weak with relatively large uncertainties, and no strong conclusions can be drawn relating to binding trends. CLR01 shows different binding trends than the other hosts. The binding is strong, and unlike the other hosts this also includes $<100 \mu \mathrm{M}$ binding of each unmethylated amino acid. ${ }^{36}$ Lysine binds with a $\mathrm{K}_{d}=30 \mu \mathrm{M}$, and the slightly more hydrophobic MML binds more strongly at $K_{d}=$ $15 \mu \mathrm{M}$. Besides this one exception, each other amino acid binds progressively weaker with increasing methylation. Unlike for all other hosts, dimethylarginines are not measurably bound by CLR01 under the conditions of the experiment.

Table 1. $K_{d}$ values determined by IDA for each host-guest complex in $10 \mathrm{mM}$ phosphate buffer. ${ }^{a}$

\begin{tabular}{|c|c|c|c|c|c|c|c|}
\hline & $\mathbf{s C x} 4^{\mathrm{b}}$ & sCx4-CHO $^{\text {b }}$ & ${\mathbf{s C x} 4-\mathrm{NO}_{2}{ }^{\mathrm{b}}}$ & M1 $^{\mathrm{c}}$ & $\mathbf{M 2}^{\mathrm{c}}$ & $\mathbf{P C}^{\mathrm{d}}$ & CLR01 $^{\mathrm{d}}$ \\
\hline & $\mathrm{K}_{\mathrm{d}}(\mu \mathrm{M})$ & $\mathrm{K}_{\mathrm{d}}(\mu \mathrm{M})$ & $\mathrm{K}_{\mathrm{d}}(\mu \mathrm{M})$ & $\mathrm{K}_{\mathrm{d}}(\mu \mathrm{M})$ & $\mathbf{\Lambda}_{d}(\mu \mathrm{N} \mathbf{1})$ & $\mathbf{K}_{\mathrm{d}}(\mu \mathrm{M})$ & $\mathrm{K}_{\mathrm{d}}(\mu \mathrm{M})$ \\
\hline Arginine & $>1500$ & $>1500$ & $>1500$ & $>1500$ & $>1500$ & $1100 \pm 630$ & $75 \pm 20$ \\
\hline MMA & $>1500$ & $>1500$ & $>1500$ & $45 \pm 10$ & $>1500$ & $1230 \pm 540$ & $135 \pm 30$ \\
\hline SDMA & $1090 \pm 300$ & $370 \pm 150$ & $>1500$ & $35 \pm 10$ & $40 \pm 15$ & $810 \pm 270$ & $>1500$ \\
\hline Lysine & $>1500$ & $>1500$ & $>1500$ & $>1500$ & $>1500$ & $>1500$ & $30 \pm 5$ \\
\hline MML & $930 \pm 120$ & $470 \pm 110$ & $610 \pm 130$ & $160 \pm 60$ & $>1500$ & $>1500$ & $15 \pm 5$ \\
\hline DML & $300 \pm 40$ & $240 \pm 50$ & $410 \pm 60$ & $70 \pm 10$ & $340 \pm 150^{\mathrm{e}}$ & $980 \pm 710$ & $30 \pm 5$ \\
\hline TML & $120 \pm 20$ & $100 \pm 30$ & $150 \pm 30$ & $15 \pm 5$ & $30 \pm 10$ & $1040 \pm 360$ & $65 \pm 20$ \\
\hline
\end{tabular}

[a] All titrations were carried out in $10 \mathrm{mM} \mathrm{NaH}_{2} \mathrm{PO}_{4}$ buffer at $\mathrm{pH}$ 7.4. See the Supporting Information for titration curves experimental details, and fitting details. All $\mathrm{K}_{\mathrm{d}}$ values arise from fits with $\mathrm{R}^{2} \geq 0.95$ except where indicated. [b] Lucigenin (LCG) was used as indicator. [c] Rhodamine 6G (R6G) was used as indicator. [d] 4(4-Diethylaminostyryl)-1-methylpyridinium iodide (4-ASP) was used as indicator. [e] $R^{2} \geq 0.92$. 
To determine the effect of a higher salt concentration, IDAs were also done in a more concentrated $50 \mathrm{mM}$ phosphate buffer with the three strongest binding hosts: M1, M2 and CLR01. We see that the strong binding and the binding trends are maintained for M2 and CLR01. The binding strength for M1 becomes 2-4fold weaker for each guest, but still remains in the "strong" range of $K_{d}$ values.

Table 2. $K_{d}$ values determined by IDA for each host-guest complex in $50 \mathrm{mM}$ phosphate buffer. ${ }^{a}$

\begin{tabular}{llll} 
& M1 $^{\text {b }}$ & M2 $^{\text {b }}$ & CLR01 $^{\mathrm{c}}$ \\
$\mathrm{K}_{\mathrm{d}}(\mu \mathrm{M})$ & $>1500$ & $\mathrm{~K}_{\mathrm{d}}(\mu \mathrm{M})$ \\
\hline Arginine & $>1500$ & $>1500$ & $93 \pm 26$ \\
MMA & $177 \pm 63$ & $124 \pm 42$ & $157 \pm 28$ \\
ADMA & $30 \pm 11$ & $42 \pm 11$ & $>1500$ \\
SDMA & $56 \pm 14$ & $>1500$ & $>1500$ \\
Lysine & $>1500$ & $>1500$ & $51 \pm 14$ \\
MML & $297 \pm 145$ & $188 \pm 112^{\mathrm{d}}$ & $33 \pm 6$ \\
DML & $205 \pm 52$ & $24 \pm 5$ & $55 \pm 9$ \\
TML & $30 \pm 6$ & $38 \pm 5$
\end{tabular}

[a] All titrations were carried out in $50 \mathrm{mM} \mathrm{NaH}_{2} \mathrm{PO}_{4}$ buffer at $\mathrm{pH}$ 7.4. See the Supporting Information for titration curves, experimental details, and fitting details. All $K_{d}$ values arise from fits with $R^{2} \geq 0.95$ except where indicated. [b] Rhodamine 6G (R6G) was used as indicator. [c] 4-(4-Diethylaminostyryl)-1methylpyridinium iodide (4-ASP) was used as indicator. [d] $R^{2} \geq 0.93$.

We did molecular modeling for six of the hosts to provide a general view of the differences and similarities between the hosts for certain key complexes. Molecular modeling was done using minimization in explicit water for all the indicated complexes using Maestro (Schrödinger, Inc). Host sCx4-CHO, sCx4-NO 2 , M1, M2, PC, CLR01 were modeled in complex with ADMA (Figure 4) and hosts M1, M2, PC, CLR01 were also modeled each in complex with MML and TML (Supporting Information) in order to gain further insight into selectivity among these guests. The models reveal the extent and nature of interactions between hosts and guests, and show qualitatively that the different hosts generally fit into two classes: open topology hosts that engage only the charged side chain of the guests (the calixarenes and PC), and closed topology hosts that almost completely surround their guests (M1, M2, and CLR01). They also reveal differences in host-guest interactions among the different host classes. The connections between these structural features and guest-binding selectivities are discussed below.

By comparing across host classes, we can derive some general lessons about the contributions from electrostatics, hydrophobicity, and topological shape matching.

While electrostatics are undoubtedly important for molecular recognition, our binding data show that they are not the key determining factor for guest selectivity. We know from other literature that neutral guests do not bind the calixarene hosts as strongly ${ }^{22}$, where the tweezer-type host do have a precedent as strong binding host. ${ }^{36,37}$ Yet, the comparison across all three host classes makes it clear that the selective binding of charged species in this relatively salty environment is not strongly controlled by the charges on hosts or guests. All guests have a zwitterionic a-amino-acid component, and a net charge of +1 . The methylated guests spread the positive charge around to more distributed regions of the guest's surface area. The calixarenes and acyclic CBs have net charges of between -4 and -5 , and yet bind guests with affinities that vary over $>2$ orders of magnitude. The clip-type hosts have net charges of between -2 and -4 (depending on the degree of second ionization of the phosphate groups), and also generate $K_{d}$ values that vary by almost 100 -fold between different guests. M1 and $\mathbf{M} 2$ have eight carbonyl groups that can form ion-dipole interaction, and two sulfonate groups that can form ion-ion interactions. Molecular modeling corroborates that the ion-dipole interactions are the main electrostatic interaction that is taking place for $\mathbf{M} 1$ and $\mathbf{M} 2$, whereas charged interactions between the guests and the hosts' sulfonate arms are not as prominently seen in the energy-minimized structures (Figure 4c-d). The calixarenes also have multiple sulfonate groups, and in these complexes host-guest salt bridges are prominent features of the complexes (Figure $4 a-b$ and Supporting Information). PC and CLR01 both have two phosphate groups that can have ion-ion interactions between the charged phosphate groups and the guanidinium, see Figure $4 \mathrm{e}-\mathrm{f}$. All of the hosts can form cation-pi interactions with their cationic guests, although the geometric details vary.

Figure 4. Molecular modelling of each host in complex with ADMA. a) sCx4-
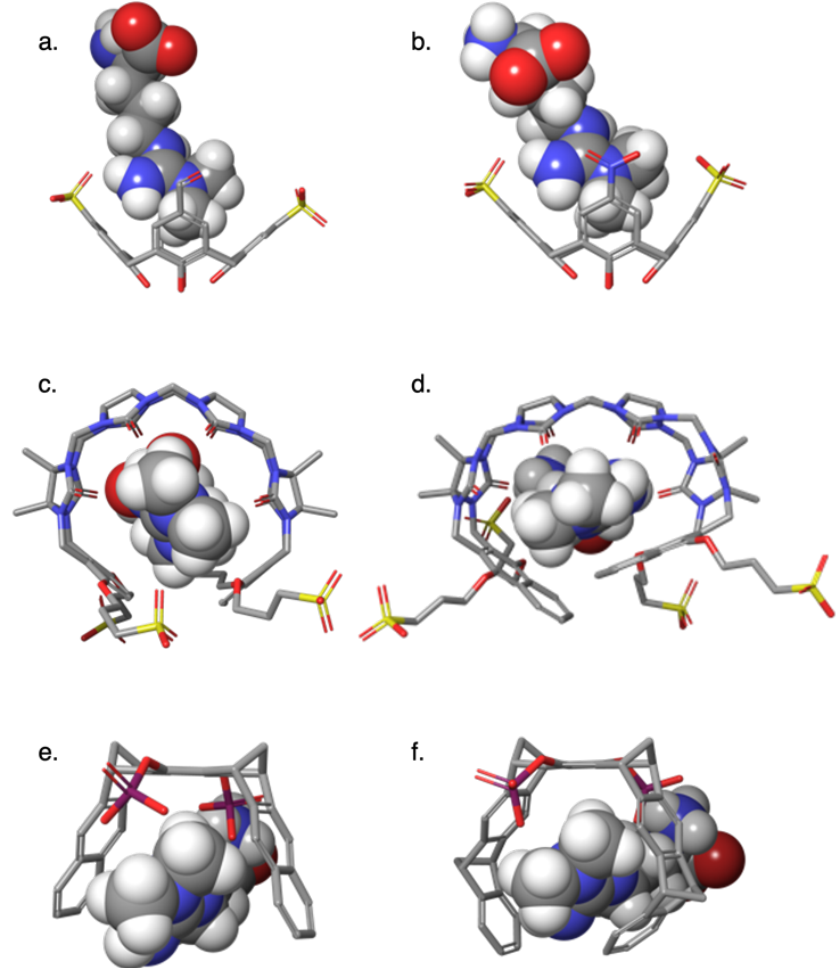

f.

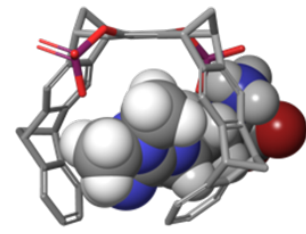

CHO, front view, b) sCx4-NO $\mathbf{N}_{2}$, front view/ c) M1, top view. d) M2, top view. e) PC, front view. f) CLR01, front view. Molecules were energy-minimized in explicit water (not shown) using OPLS_2005 as implemented in Maestro (Schödinger, Inc). See Supporting information for more views and for other hostguest complexes.

Guest hydrophobicity is the main determinant for guest selectivity in hosts with more open topologies, including all calixarenes and PC. Methylation of arginine and lysine increases the volume of the head groups. This results in an increased hydrophobic surface area, a change to a more diffuse charge distribution, and decrease in the guest's potential to form strong $\mathrm{NH}$ hydrogen bonds. ${ }^{38}$ This modification is favourable when looking at the calixarenes and PC, where an increased number of methyl groups results in a stronger binding. This trend can clearly be observed for the lysine guests, where unmethylated lysine displays weak binding and TML displays strong binding to the hosts (Table 1). When looking at the dimethylated arginines we also see that position of the methyl groups has an influence. The models show that the hydrophobic surface area of ADMA is 
localized in one patch made up of two geminal methyl groups, whereas the hydrophobic surface area of SDMA is separated with methyls on two distal nitrogen atoms. In open-topology hosts that don't constrain for molecular shape, this results in a stronger engagement of ADMA in the host pockets relative to SDMA.

Topological shape matching also contributes to the binding strengths and selectivity of the host-guest complexes. The relative openness of topology and is a key determinant. ${ }^{39}$ A shallower or open binding pocket is unfavourable for binding the free amino acids, as would be expected from the arguments made above. This can be clearly observed for the calixarenes and PC, where there isn't a large complementary overlap of the surface area, the binding is weak. Hosts with closed topologies can display selectivities that run against the underlying trends caused by hydrophobicity. In general, increasing methylation increases the tendency to bind, but binding can be discouraged when shape and fit are incompatible. This can most clearly be observed for CLR01 and M2. CLR01 is in the middle of the "openness range" of this library, it has nine rings forming its tweezer shape. Its binding pocket is a perfect fit to bind medium-sized hydrophobic molecules and is the only host in this library with strong binding for both unmethylated arginine and lysine. When looking at the dimethylated arginines, ADMA's geminal methyls can fit into the pocket of most hosts (see Figure 4). The exception is M2, where ADMA binding is 3-fold weaker compared to SDMA. This makes M2 a very rare example of an SDMA-selective host molecule. The structural difference between $\mathbf{M} 1$ and $\mathbf{M} 2$ is the naphthalene of M2. This creates a bigger, more hydrophobic, and somewhat more closed-off binding pocket. This small change makes a significant difference in the binding properties of the host-guest complexes. When looking at MML versus TML we can see that M1 keeps the same formation where M2 must accommodate for its bigger structure and is slightly askew (see Supporting Information). PC and CLR01 are more rigid molecules, and do not have the option to flex to accommodate guests in the same way as the acyclic cucurbiturils.

\section{Conclusion}

In conclusion, we have studied a host library containing calixarenes, acyclic cucurbiturils, and cleft-like hosts. From a series of guest binding studies with methylated amino acids we see that the relatively open hosts, calixarenes and PC, have an overall weaker binding than the host with a deeper binding pocket. We found that the CLR01 host was the best size for the nonmethylated amino acids, showing a strong binding for arginine and lysine which the other hosts do not. The methylated arginine guests are bound the strongest by the acyclic CB hosts, but in a weaker range of $\mathrm{K}_{d}$ values the host $\mathbf{s} \mathbf{C} \times \mathbf{4}-\mathrm{NO}_{2}$ shows good selectivity for ADMA over all other arginines. TML is bound strongly by all hosts, except PC, but is only bound with good selectivity over other related guests by M2. While this study is fundamental and not targeted at applied science, we do identify useful new selectivities, such as the complete selectivity of CLR01 for methyllysines over methylarginines, and the novel selectivity for SDMA over ADMA that is displayed by M2. This study also allows us to see some interesting trends emerge from direct comparison of different host classes under identical conditions. For example, in most ways PC behaves more like the sCx4 hosts than like its close chemical relative CLR01. In this work we can tie that similar behaviour across many guests to similarities in host topology that mostly override the more obvious differences in functional group identity and arrangement that typically dominate our thinking about host-guest binding. We think that additional non-dogmatic, collaborative, open comparisons of host molecules will lead to more such insights in the near future.

\section{Acknowledgements}

We thank Hendrik Kirschner and Christine Beuck for carrying out preliminary studies. We thank Rebecca Hof for the training and Allison Selinger for helpful discussions on the data analysis for indicator displacement assays.

\section{Funding}

We thank the National Science Foundation (CHE-1807486), NSERC (RGPIN-2019-04806), National Science Foundation (CHE-1807486), Deutsche Forschungsgemeinschaft (DFG) to TS (Schr 604/19-1) and PB (BA1624/15-1) for their financial support.

Keywords: macrocycles $\cdot$ methylated amino acids $\cdot$ host-guest chemistry $\bullet$ indicator displacement assays

(1) Beatty, M. A.; Hof, F. Host-guest binding in water, salty water, and biofluids: general lessons for synthetic, bio-targeted molecular recognition. Chem Soc Rev 2021.

(2) Schlesinger, S.; Sonntag, S. R.; Lieb, W.; Maas, R. Asymmetric and Symmetric Dimethylarginine as Risk Markers for Total Mortality and Cardiovascular Outcomes: A Systematic Review and Meta-Analysis of Prospective Studies. PLoS One 2016, 11, e0165811.

(3) DeBerardinis, R. J.; Thompson, C. B. Cellular metabolism and disease: what do metabolic outliers teach us? Cell 2012, 148, 1132-1144.

(4) Gowda, G. A.; Zhang, S.; Gu, H.; Asiago, V.; Shanaiah, N.; Raftery, D. Metabolomics-based methods for early disease diagnostics. Expert Rev Mol Diagn 2008, 8, 617-633.

(5) Ruigrok, V. J.; Levisson, M.; Eppink, M. H.; Smidt, H.; van der Oost, J. Alternative affinity tools: more attractive than antibodies? Biochem J 2011, 436, 1-13.

(6) Egelhofer, T. A.; Minoda, A.; Klugman, S.; Lee, K.; Kolasinska-Zwierz, P.; Alekseyenko, A. A.; Cheung, M. S.; Day, D. S.; Gadel, S.; Gorchakov, A. A.; Gu, T.; Kharchenko, P. V.; Kuan, S.; Latorre, I.; Linder-Basso, D.; Luu, Y.; Ngo, Q.; Perry, M.;

Rechtsteiner, A.; Riddle, N. C.; Schwartz, Y. B.; Shanower, G. A.; Vielle, A.; Ahringer, J.; Elgin, S. C.; Kuroda, M. I.; Pirrotta, V.; Ren, B.; Strome, S.; Park, P. J.; Karpen, G. H.; Hawkins, R. D.; Lieb, J. D. An assessment of histone-modification antibody quality. Nat Struct Mol Biol 2011, 18, 91-93.

(7) Kungulovski, G.; Jeltsch, A. Quality of histone modification antibodies undermines chromatin biology research. F1000Res 2015, 4, 1160.

(8) Rothbart, S. B.; Dickson, B. M.; Raab, J. R.; Grzybowski, A. T.; Krajewski, K.; Guo, A. H.; Shanle, E. K.; Josefowicz, S. Z.; Fuchs, S. M.; Allis, C. D.; Magnuson, T. R.; Ruthenburg, A. J.; Strahl, B. D. An Interactive Database for the Assessment of Histone Antibody Specificity. Mol Cell 2015, 59, 502-511.

(9) Acharya, P.; Quinlan, A.; Neumeister, V. The ABCs of finding a good antibody: How to find a good antibody, validate it, and publish meaningful data. F1000Res 2017, 6, 851.

(10) Martins, J. N.; Lima, J. C.; Basilio, N. Selective Recognition of Amino Acids and Peptides by Small Supramolecular Receptors. Molecules 2020, 26. 
(11) Shinkai, S.; Araki, K.; Manabe, O. Does the Calixarene Cavity Recognize the Size of Guest Molecules - on the Hole-Size Selectivity in Water-Soluble Calixarenes. J Chem Soc Chem Comm 1988, 187-189.

(12) Zhang, B.; Isaacs, L. Acyclic Cucurbit[n]uril-type Molecular Containers: Influence of Aromatic Walls on their Function as Solubilizing Excipients for Insoluble Drugs. J Med Chem 2014, 57, 9554-9563.

(13) Hadrovic, I.; Rebmann, P.; Klarner, F. G.; Bitan, G.; Schrader, T. Molecular Lysine Tweezers Counteract Aberrant Protein Aggregation. Front Chem 2019, 7, 657.

(14) Talbiersky, P.; Bastkowski, F.; Klarner, F. G.; Schrader, T. Molecular clip and tweezer introduce new mechanisms of enzyme inhibition. J Am Chem Soc 2008, 130, 9824-9828.

(15) Cooke, J. P. ADMA: its role in vascular disease. Vasc Med 2005, 10, S11-S17.

(16) Cooke, J. P.; Ghebremariam, Y. T. DDAH says NO to ADMA. Arterioscler Thromb Vasc Biol 2011, 31, 1462-1464.

(17) Tsikas, D.; Bollenbach, A.; Hanff, E.; Kayacelebi, A. A Asymmetric dimethylarginine (ADMA), symmetric dimethylarginine (SDMA) and homoarginine (hArg): the ADMA, SDMA and hArg paradoxes. Cardiovascular diabetology 2018, 17, 1.

(18) Emrich, I. E.; Zawada, A. M.; Martens-Lobenhoffer, J.; Fliser, D.; Wagenpfeil, S.; Heine, G. H.; Bode-Boger, S. M. Symmetric dimethylarginine (SDMA) outperforms asymmetric dimethylarginine (ADMA) and other methylarginines as predictor of renal and cardiovascular outcome in non-dialysis chronic kidney disease. Clin Res Cardiol 2018, 107, 201-213.

(19) Rowe, E. M.; Xing, V.; Biggar, K. K. Lysine methylation: Implications in neurodegenerative disease. Brain Res 2019, 1707, 164-171.

(20) Hamamoto, R.; Saloura, V.; Nakamura, Y. Critical roles of non-histone protein lysine methylation in human tumorigenesis. Nat Rev Cancer 2015, 15, 110-124.

(21) Daze, K. D.; Pinter, T.; Beshara, C. S.; Ibraheem, A.; Minaker, S. A.; Ma, M. C. F.; Courtemanche, R. J. M.; Campbell, R. E.; Hof, F. Supramolecular hosts that recognize methyllysines and disrupt the interaction between a modified histone tail and its epigenetic reader protein. Chem Sci 2012, 3, 2695-2699.

(22) Beshara, C. S.; Jones, C. E.; Daze, K. D.; Lilgert, B. J.; Hof, F. A Simple Calixarene Recognizes Post-translationally Methylated Lysine. Chembiochem 2010, 11, 63-66.

(23) Lindsey A. Ingerman; Cuellar, M. E.; Waters, M. L. A small molecule receptor that selectively recognizes trimethyl lysine in a histone peptide with native protein-like affinity. Chem. Commun. 2010, 46, 1839-1841.

(24) Gruber, T. Synthetic Receptors for the Recognition and Discrimination of Post-Translationally Methylated Lysines.

Chembiochem 2018, 19, 2324-2340.

(25) Mullins, A. G.; Pinkin, N. K.; Hardin, J. A.; Waters, M. L. Achieving High Affinity and Selectivity for Asymmetric Dimethylarginine by Putting a Lid on a Box. Angew Chem Int Ed Engl 2019, 58, 5282-5285.

(26) James, L. I.; Beaver, J. E.; Rice, N. W.; Waters, M. L. A synthetic receptor for asymmetric dimethyl arginine. J. Am. Chem. Soc. 2013, 135, 6450-6455.

(27) Bode-Boger, S. M.; Scalera, F.; Ignarro, L. J. The Larginine paradox: Importance of the L-arginine/asymmetrical dimethylarginine ratio. Pharmacology \& therapeutics 2007, 114, 295306.

(28) Adler-Abramovich, L.; Vaks, L.; Carny, O.; Trudler, D.; Magno, A.; Caflisch, A.; Frenkel, D.; Gazit, E. Phenylalanine assembly into toxic fibrils suggests amyloid etiology in phenylketonuria. Nat Chem Biol 2012, 8, 701-706.

(29) Blackburn, P. R.; Gass, J. M.; Vairo, F. P. E.; Farnham, K. M.; Atwal, H. K.; Macklin, S.; Klee, E. W.; Atwal, P. S. Maple syrup urine disease: mechanisms and management. Appl Clin Genet 2017, 10, 57-66.

(30) McQuinn, K.; Mclndoe, L. S.; Hof, F. Insights into the post-translational methylation of arginine from studies of guanidinium-water nanodroplets. Chem-Eur J 2008, 14, 6483-6489.

(31) Dsouza, R. N.; Pischel, U.; Nau, W. M. Fluorescent Dyes and Their Supramolecular Host/Guest Complexes with Macrocycles in Aqueous Solution. Chemical Reviews 2011, 111, 7941-7980.
(32) Warmerdam, Z.; Kamba, B. E.; Shaurya, A.; Sun, X. X.; Maguire, M. K.; Hof, F. Calix[4]arene sulfonate hosts selectively modified on the upper rim: a study of nicotine binding strength and geometry. Supramol Chem 2021

(33) Ma, D.; Zhang, B.; Hoffmann, U.; Sundrup, M. G.; Eikermann, M.; Isaacs, L. Acyclic cucurbit[n]uril-type molecular containers bind neuromuscular blocking agents in vitro and reverse neuromuscular block in vivo. Angew Chem Int Ed Engl 2012, 51, 11358-11362.

(34) Ma, D.; Zavalij, P. Y.; Isaacs, L. Acyclic cucurbit[n]uril congeners are high affinity hosts. J Org Chem 2010, 75, 4786-4795.

(35) Cao, L.; Sekutor, M.; Zavalij, P. Y.; Mlinaric-Majerski, K.; Glaser, R.; Isaacs, L. Cucurbit[7]urilguest pair with an attomolar dissociation constant. Angew Chem Int Ed Engl 2014, 53, 988-993.

(36) Fokkens, M.; Schrader, T.; Klarner, F. G. A molecular tweezer for lysine and arginine. J Am Chem Soc 2005, 127, 1441514421.

(37) Ndendjio, S. A. Z.; Isaacs, L. Molecular recognition properties of acyclic cucurbiturils toward amino acids, peptides, and a protein. Supramol Chem 2019, 31, 432-442.

(38) Evich, M.; Stroeva, E.; Zheng, Y. G.; Germann, M. W. Effect of methylation on the side-chain pKa value of arginine. Protein Sci 2016, 25, 479-486.

(39) Beaver, J. E.; Peacor, B. C.; Bain, J. V.; James, L. I. Waters, M. L. Contributions of pocket depth and electrostatic interactions to affinity and selectivity of receptors for methylated lysine in water. Org Biomol Chem 2015, 13, 3220-3226. 


\section{Entry for the Table of Contents}

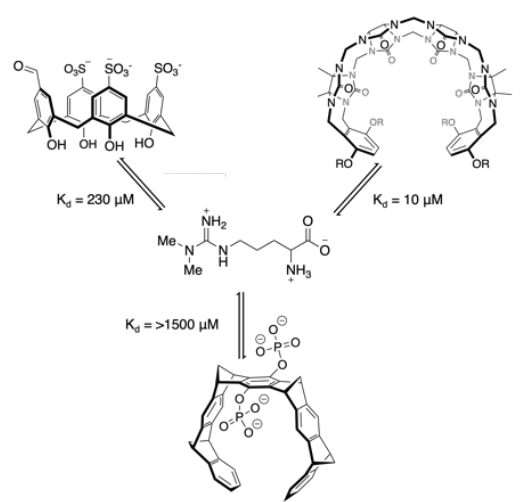

We present comparative binding studies for three different supramolecular host classes (calixarenes, acyclic cucurbiturils and cleftlike hosts), each studied with multiple methylated arginines and lysines to determine fundamental structure-function relationships. Molecular modeling was used to give an insight on the preferences of the different hosts. 


\section{Supporting information}

for

Binding methylarginines and methyllysines as free amino acids: a comparative study of multiple supramolecular host classes

\section{Table of Contents}

\section{General information and materials 2}

2. IDA titrations $\quad 2$

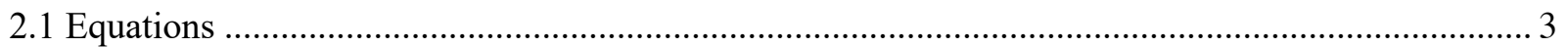

2.1.1. Outliers 3

2.1.2. The standard error $\quad 3$

2.1.3. The total standard error $\quad 3$

2.1.4. Curve fit for the direct titration 3

2.1.5. Curve fit for the competitive titration 4

2.2. Fluorescence based studies $10 \mathrm{mM}$ buffer............................................................................. 5

2.2.1. Fluorescence based studies of $\mathbf{s} \mathbf{C x} 4$

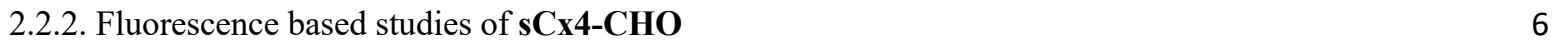

$\begin{array}{ll}\text { 2.2.3. Fluorescence based studies of } \mathbf{s C x} 4-\mathrm{NO2} & 7\end{array}$

2.2.4. Fluorescence based studies of CLR01 1

2.2.5. Fluorescence based studies of PC 9

2.2.6. Fluorescence based studies of M1 10

2.2.7. Fluorescence based studies of $\mathbf{M 2} \quad 11$

2.3. Fluorescence based studies $50 \mathrm{mM}$ buffer 2.3.1. Fluorescence based studies of $\mathbf{M 1}$.................... 12

2.3.2. Fluorescence based studies of $\mathbf{M} 2$

2.3.4. Fluorescence based studies of CLR01 14

3. Molecular Modeling 15

3.1. Each host modeled with ADMA. Front, back, top, and bottom views for all complexes.............. 15

3.2. Hosts M1, M2, PC, CLR01 each modeled with MML and with TML...................................... 18

4. References 
1. General information and materials

The host used in these studies have all been previously published or are commercially available. sCx4 (4sulfocalic[4]arene Hydrate) was purchased from Tokyo Chemical Industry (TCI) (CAS 112269-92-8, $>94.0 \%$ ). sCx4-CHO ${ }^{1}$, sCx4-NO2 $^{2}, \mathbf{M 1}^{3}, \mathbf{M 2}^{3}, \mathbf{C L R O 1}^{4}$ and $\mathbf{P C}^{5}$ have been previously published. The dyes used in these studies have been purchased from Sigma-Aldrich, lucigenin (CAS 2315-97-1), 4-ASP (CAS 68971-03-9, 98\%), R6G (CAS 989-38-8, 95 \%). The guests were purchased from different suppliers. Arginine was purchased from Calbiochem (CAS 1119-34-2, 99.7\%). Lysine was purchased from

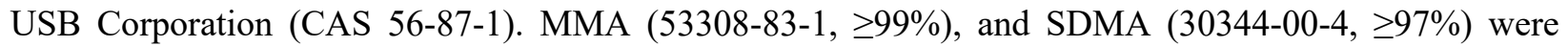
purchased from ENZO. ADMA (CAS 220805-22-1) and MML (CAS 7622-29-9) were purchased from

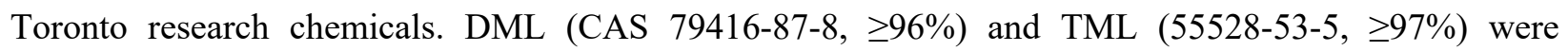
purchased from Sigma-Aldrich.

2. IDA titrations

The IDAs were conducted in 384 well plates (Nunc ${ }^{\mathrm{TM}}$ 384-Well, Non-Treated, Flat-Bottom Microplate). The fluorescent signal was read on the Cytation 5 (Software Version 3.05.11) at room temperature. All wells had a final total volume of $50 \mu \mathrm{L}$. The IDA data was analysed in GraphPad Prism Version 8.3.0 (328). One exemplary replica is shown for each host. All of the experiments for the $10 \mathrm{mM}$ phosphate buffer were done as duplicates of triplicates. All of the experiments for the $50 \mathrm{mM}$ phosphate buffer were done in triplicate.

The fluorescent signal for the calix[4]arene host titrations was read as a fluorescence endpoint measurement. Lucigenin $(0.25 \mu \mathrm{M})$ was used as the indicator. The settings were as follows: Excitation: 369/20, Emission: 475/20. Optics: Top, Gain: extended. Light Source: Xenon Flash, Lamp Energy: High, Extended Dynamic Range. Read Speed: Normal, Delay: $100 \mathrm{msec}$, Measurements/Data Point: 10. Read Height: $10.5 \mathrm{~mm}$.

The fluorescent signal for CLR01 and PC titrations was read as a fluorescence endpoint measurement. 4$\operatorname{ASP}(0.20 \mu \mathrm{M})$ was used as the indicator. The settings were as follows: Excitation: 490/10, Emission: 610/10. Optics: Bottom, Gain: extended. Light Source: Xenon Flash, Lamp Energy: High, Extended Dynamic Range. Read Speed: Normal, Delay: 100 msec, Measurements/Data Point: 10. Read Height: 10.5 $\mathrm{mm}$.

The fluorescent signal for M1 and M2 titrations was read as a fluorescence endpoint measurement. R6G $(0.10 \mu \mathrm{M})$ was used as the indicator. The settings were as follows: Excitation: 510/10, Emission: 550/10. Optics: Bottom, Gain: extended. Light Source: Xenon Flash, Lamp Energy: High, Extended Dynamic Range. Read Speed: Normal, Delay: $100 \mathrm{msec}$, Measurements/Data Point: 10. Read Height: 10.5 mm. 


\subsection{Equations}

\subsubsection{Outliers}

Outliers are determined by the Dixon's Q-test, Equation 1, where the gap is the absolute difference between the outlier in question and the closest number to it. With three observations and at $95 \%$ confidence, $\mathrm{Q}>$ $0.970=\mathrm{Q}_{95 \%, \mathrm{n}=3}$, we conclude the data point is an outlier.

Equation 1. Dixon's Q-test.

$$
Q=\frac{g a p}{\text { range }}
$$

2.1.2. The standard error

The standard error is calculated to each triplicate, Equation 2.

Equation 2. Standard error.

$$
S D_{K_{i}}=\left(\ln \ln (10) * 10^{\log K_{i}}\right) * S D_{\log K_{i}}
$$

$\mathrm{SD}_{\mathrm{Ki}}=$ Standard error of $\mathrm{K}_{\mathrm{i}}$

$\log \mathrm{Ki}=$ Value of the $\log$

$\mathrm{SD}_{\log K i}=$ Standard error of the $\log \mathrm{Ki}$

2.1.3. The total standard error

The total standard error between two the triplicates is calculated by Equation 3 .

Equation 3. Total standard error.

$$
S D_{\text {total }}=\sqrt{\frac{\left(S D_{1}^{2}+S D_{2}^{2}\right)}{n}}
$$

$\mathrm{SD}_{\text {total }}=$ Standard derivation of both experiments

$\mathrm{SD}_{1}=$ Standard derivation of the first triplicate

$\mathrm{SD}_{2}=$ Standard derivation of the second triplicate

$\mathrm{n} \quad=$ Number of experiments

2.1.4. Curve fit for the direct titration

To curve fit for the direct titration Equation 4, Equation 5 are used.

Equation 4. Curve fit for the direct titration for a turn on signal.

$$
F=F_{\text {min }}+\frac{\left(F_{\max }-F_{\text {min }}\right) *\left([D]+[H]+K_{d}\right)-\sqrt{\left([D]+[H]+K_{d}\right)^{2}-4 *[H] *[D]}}{2 *[D]}
$$

Equation 5. Curve fit for the direct titration for a turn off signal

$$
F=F_{\text {max }}-\frac{\left(F_{\max }-F_{\min }\right) *\left([D]+[H]+K_{d}\right)-\sqrt{\left([D]+[H]+K_{d}\right)^{2}-4 *[H] *[D]}}{2 *[D]}
$$


$\mathrm{F} \quad=$ Fitted data point

$\mathrm{F}_{\max } \quad=$ Maximum signal

$\mathrm{F}_{\min } \quad=$ Minimum signal

[D] $=$ Molar concentration of dye in $\mu \mathrm{M}$

[H] = Molar concentration of host (titrant)

$\mathrm{K}_{\mathrm{d}} \quad=$ Dissociation constant

2.1.5. Curve fit for the competitive titration

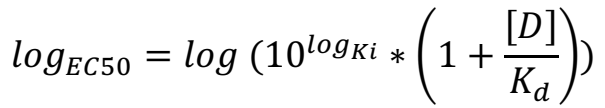

$$
\begin{aligned}
& F=F_{\min }+\left(F_{\max }-F_{\min }\right) /\left(1+10^{\left(X-\log _{E C 50)}\right)}\right.
\end{aligned}
$$

$\log _{\mathrm{EC} 50}=\log$ of the concentration of the competitor binding half-way between $\mathrm{F}_{\min }$ and $\mathrm{F}_{\max }$

$\mathrm{K}_{\mathrm{i}} \quad=$ Equilibrium dissociation constant in Molar

[D] = Concentration of dye in $\mathrm{nM}$

$\mathrm{K}_{\mathrm{d}} \quad=$ Equilibrium dissociation constant of the direct titration

$\mathrm{F} \quad=$ Fitted data point

$\mathrm{F}_{\max } \quad=$ Maximum signal

$\mathrm{F}_{\min } \quad=$ Minimum signal 


\subsection{Fluorescence based studies $10 \mathrm{mM}$ buffer}

\subsubsection{Fluorescence based studies of $\mathbf{s} \mathbf{C x} 4$}

a.

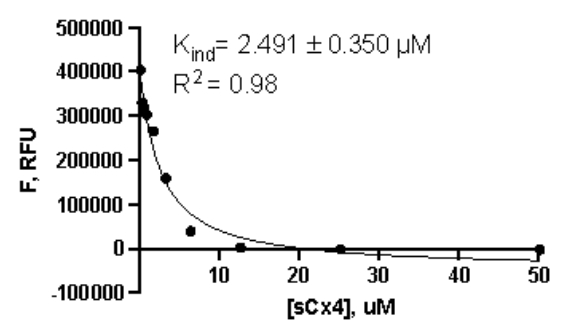

b.
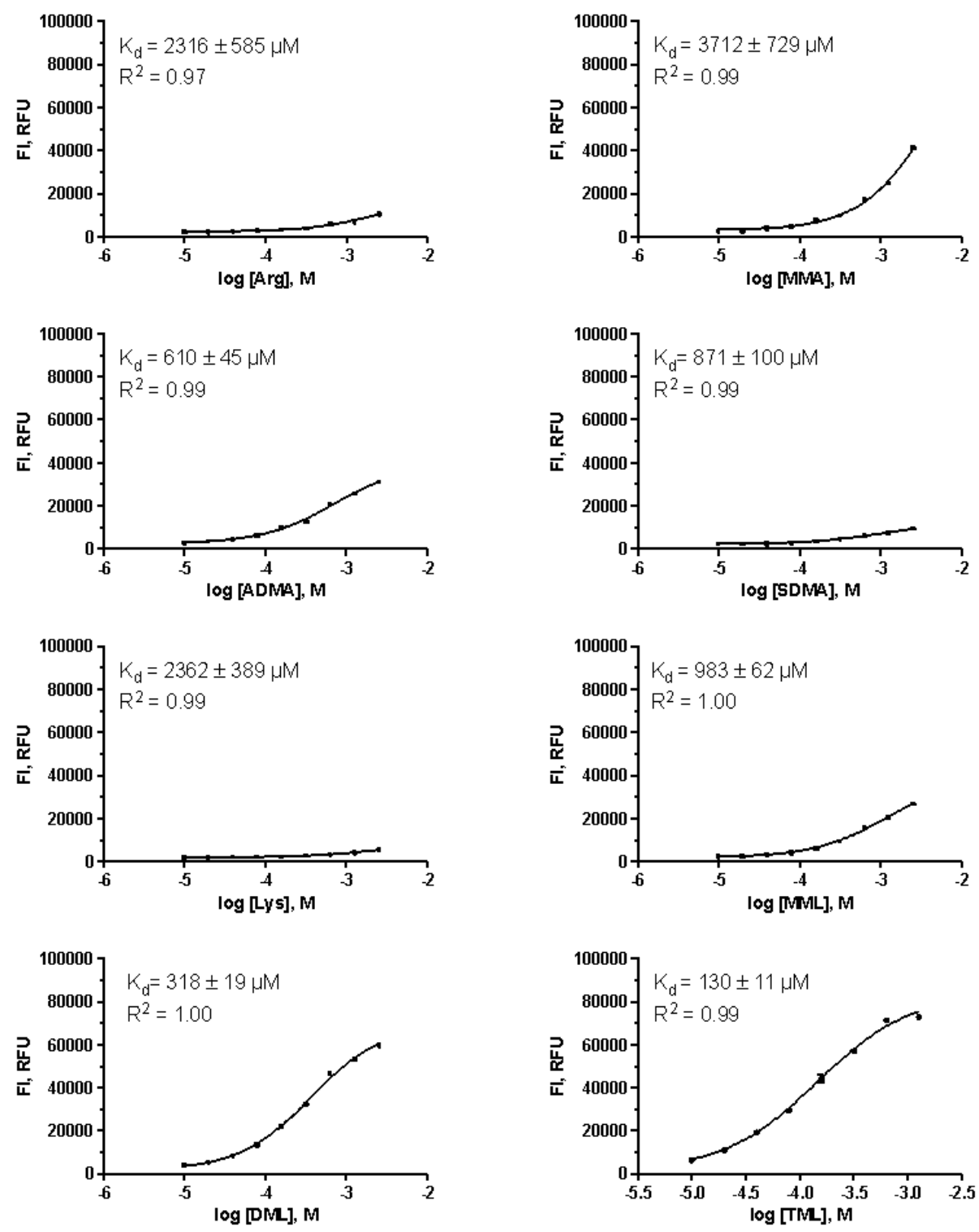

Figure S1: Fluorescence based studies of sCx4. a) Direct titration of LCG $(0.25 \mu \mathrm{M})$ with sCx4 $(0-50$ $\mu \mathrm{M})$. b) Competitive titrations of Arginine, MMA, ADMA, SDMA, Lysine, MML, DML and TML (0 -

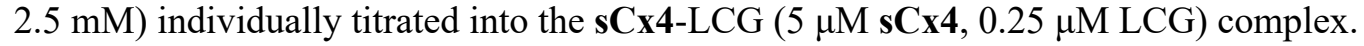




\subsubsection{Fluorescence based studies of $\mathbf{s} \mathbf{C x} 4-\mathbf{C H O}$}

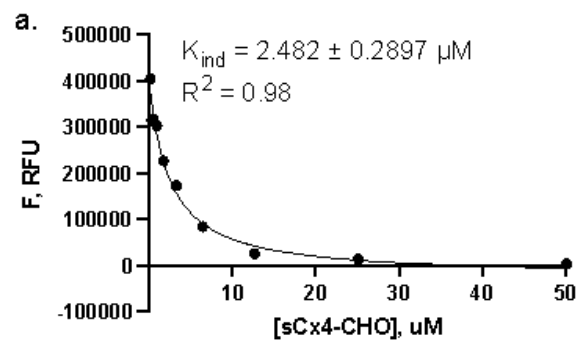

b.
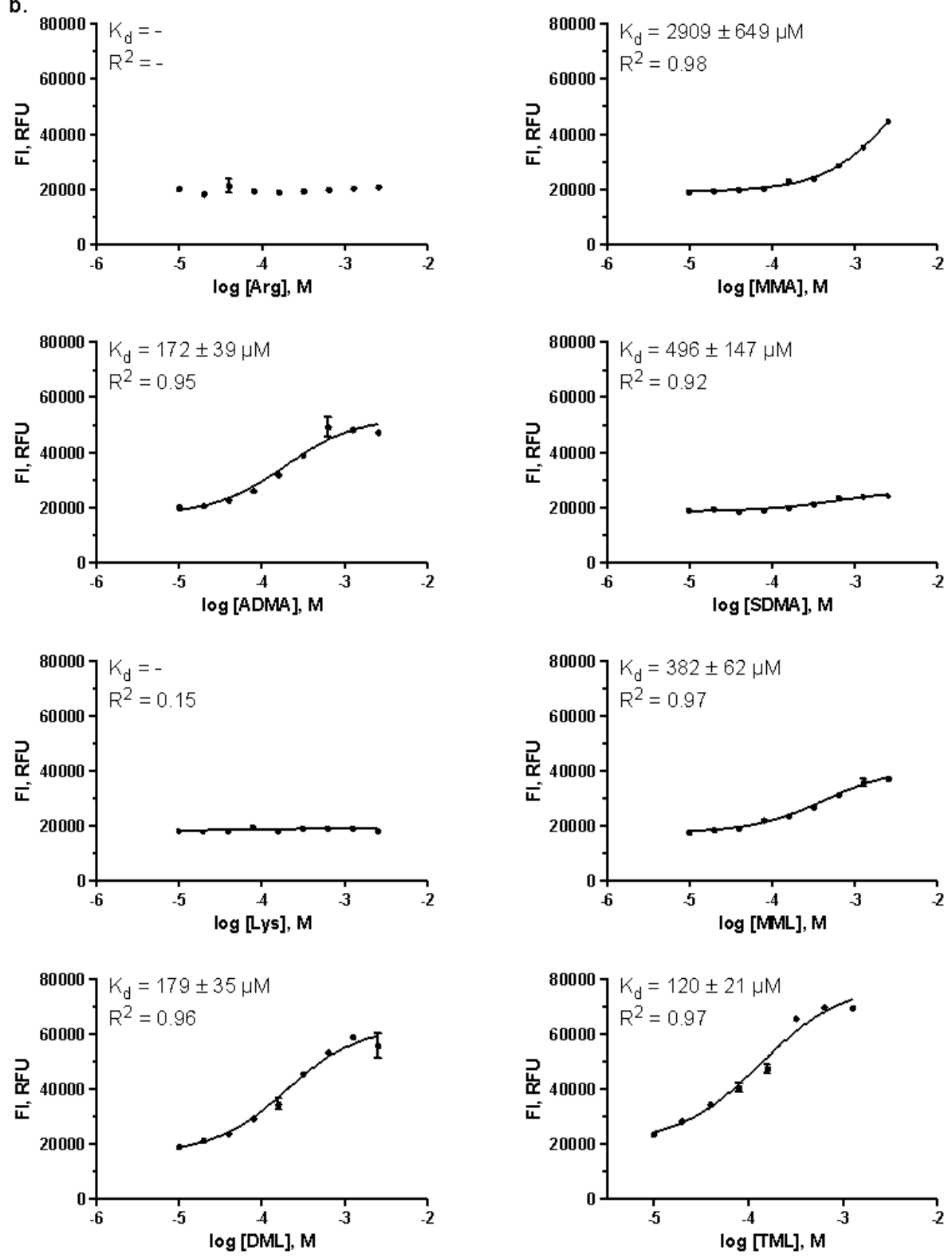

Figure S2: Fluorescence based studies of sCx4-CHO. a) Direct titration of LCG $(0.25 \mu \mathrm{M})$ with sCx4CHO $(0-50 \mu \mathrm{M})$. b) Competitive titrations of Arginine, MMA, ADMA, SDMA, Lysine, MML, DML and TML $(0-2.5 \mathrm{mM})$ individually titrated into the sCx4-CHO-LCG $(5 \mu \mathrm{M} \mathbf{s C x} 4-\mathbf{C H O}, 0.25 \mu \mathrm{M}$ LCG $)$ complex. 


\subsubsection{Fluorescence based studies of $\mathbf{s C x} 4-\mathrm{NO} 2$}
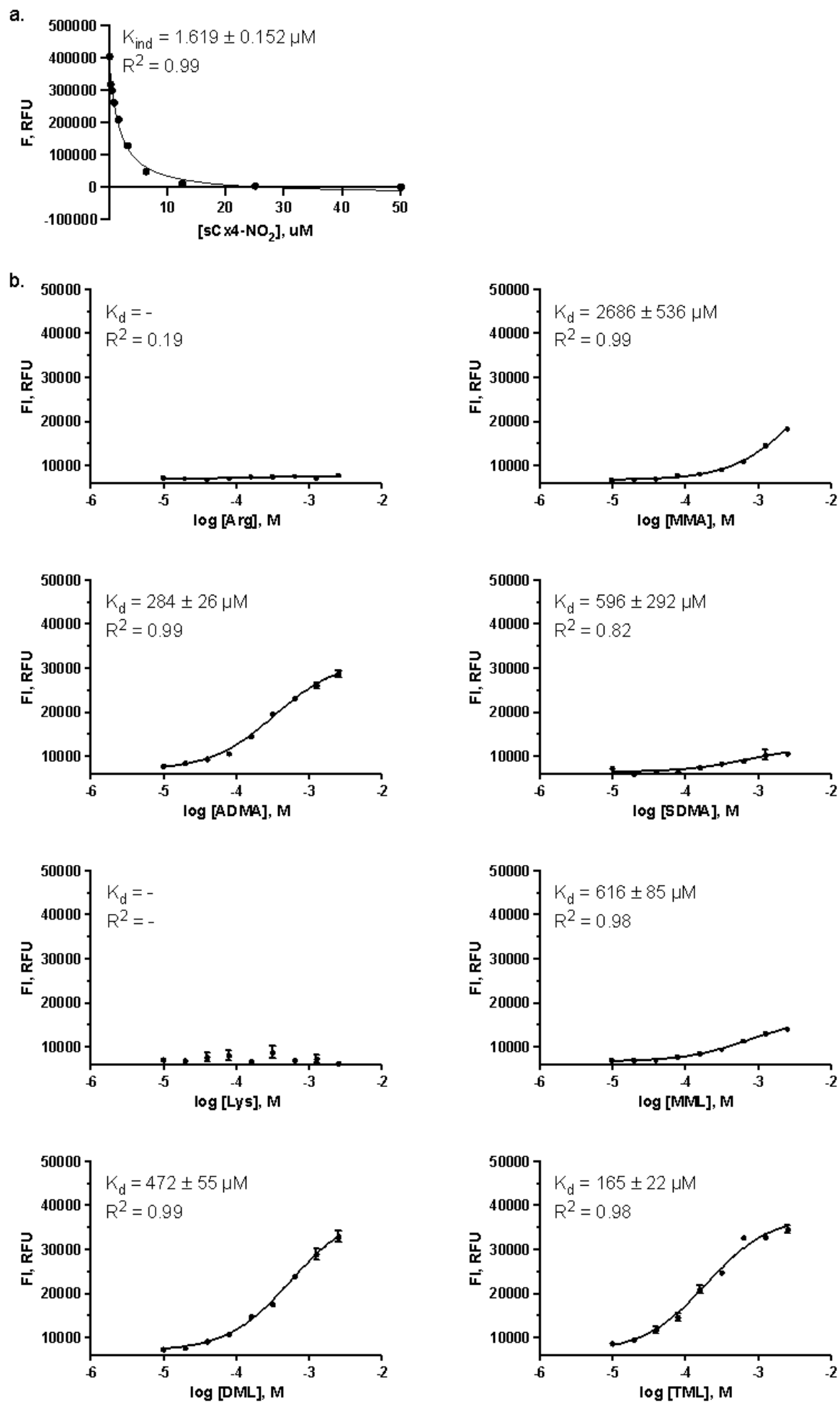

Figure S3: Fluorescence based studies of sCx4-NO2. a) Direct titration of LCG $(0.25 \mu \mathrm{M})$ with sCx4NO2 $(0-50 \mu \mathrm{M})$. b) Competitive titrations of Arginine, MMA, ADMA, SDMA, Lysine, MML, DML and TML $(0-2.5 \mathrm{mM})$ individually titrated into the sCx4-NO2-LCG $(5 \mu \mathrm{M} \mathrm{sCx4-NO2,} 0.25 \mu \mathrm{M} \mathrm{LCG})$ complex. 


\subsubsection{Fluorescence based studies of CLR01}

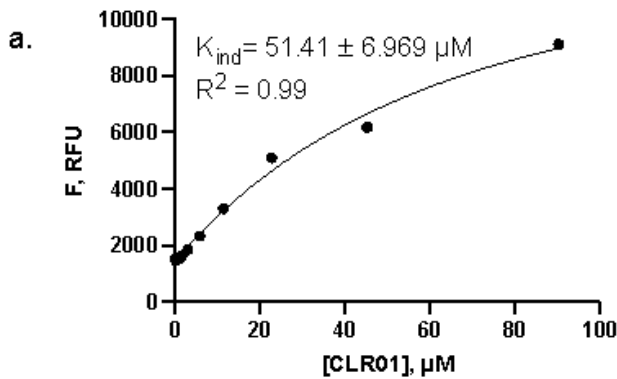

b.
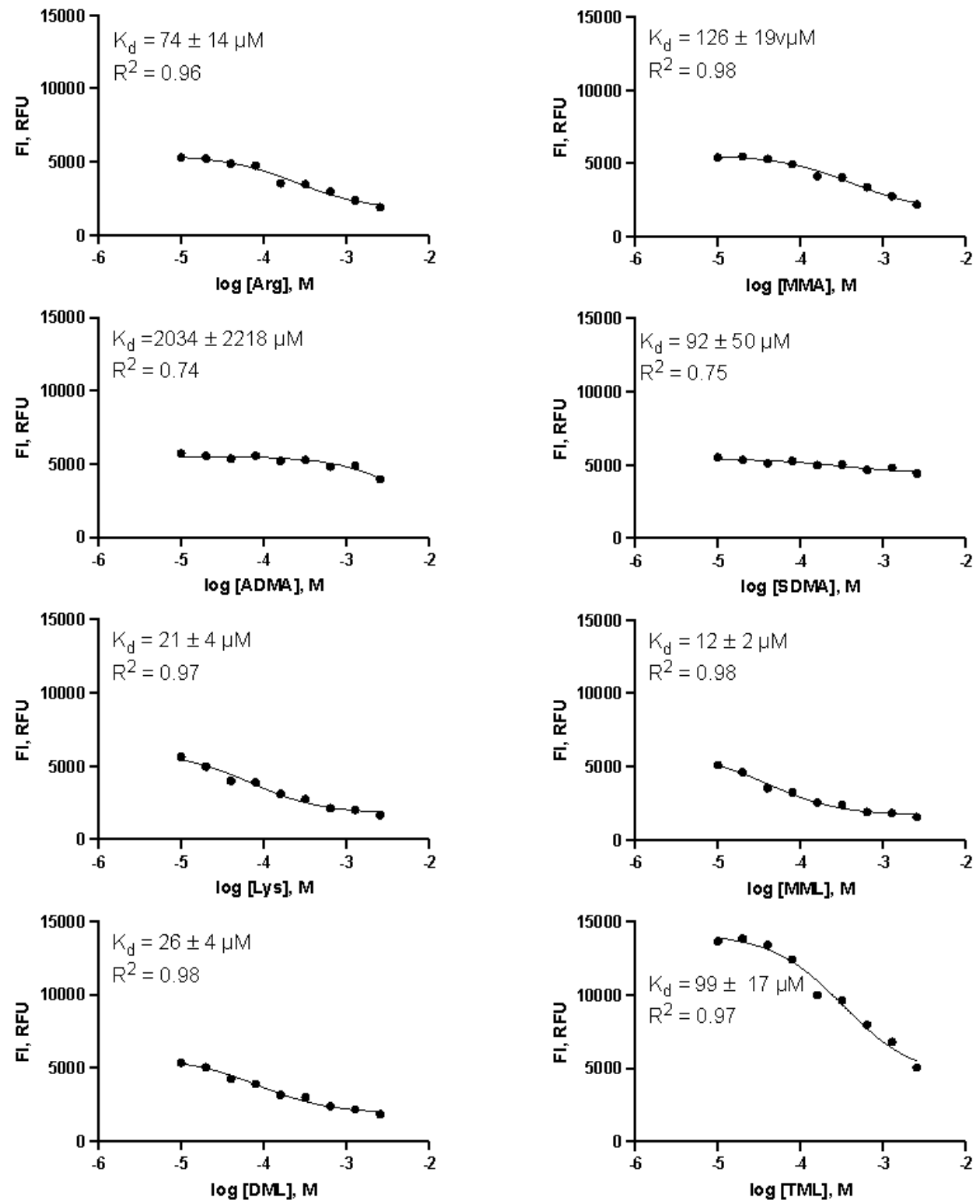

Figure S4: Fluorescence based studies of CLR01. a) Direct titration of 4-ASP $(20 \mu \mathrm{M})$ with CLR01 $(0-$ $50 \mu \mathrm{M})$. b) Competitive titrations between Arginine, MMA, ADMA, SDMA, Lysine, MML, DML and TML $(0-2.5 \mathrm{mM})$ individually titrated into the CLR01-4-ASP (70 $\mu \mathrm{M}$ CLR01, $20 \mu \mathrm{M}$ 4-ASP) complex. 


\subsubsection{Fluorescence based studies of $\mathbf{P C}$}

a.

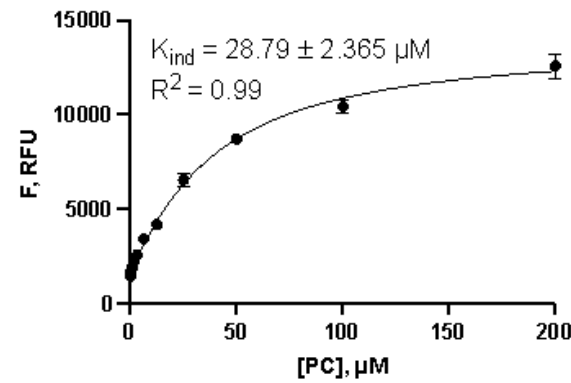

b.
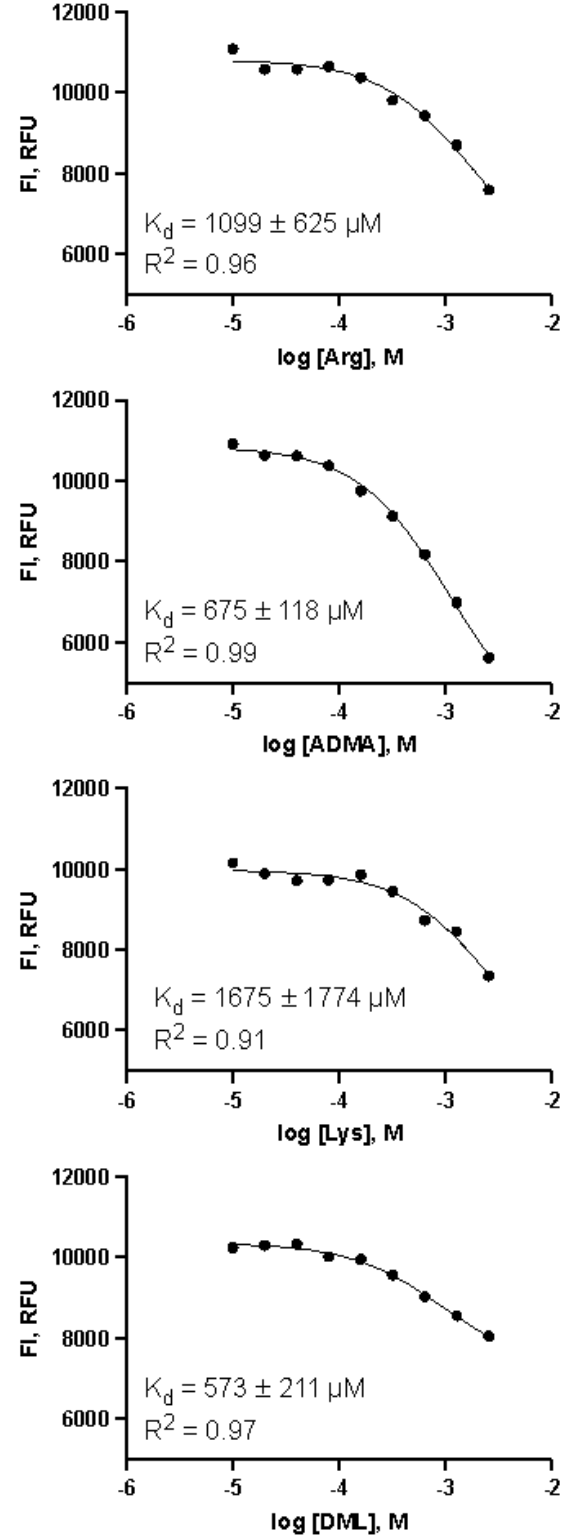
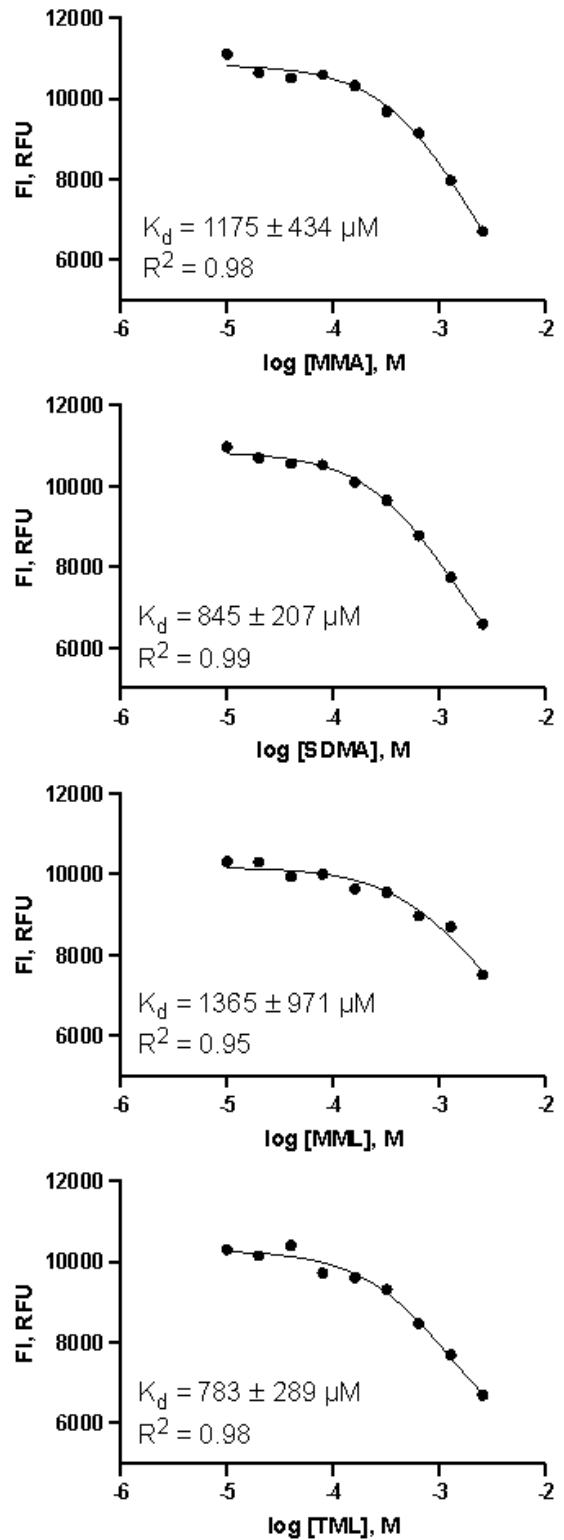

Figure S5: Fluorescence based studies of PC. a) Direct titration of 4-ASP $(20 \mu \mathrm{M})$ with PC $(0-200 \mu \mathrm{M})$. b) Competitive titrations between Arginine, MMA, ADMA, SDMA, Lysine, MML, DML and TML (0 $2.5 \mathrm{mM}$ ) individually titrated into the PC-4-ASP (30 $\mu \mathrm{M}$ PC, $20 \mu \mathrm{M}$ 4-ASP) complex. 


\subsubsection{Fluorescence based studies of $\mathbf{M 1}$}
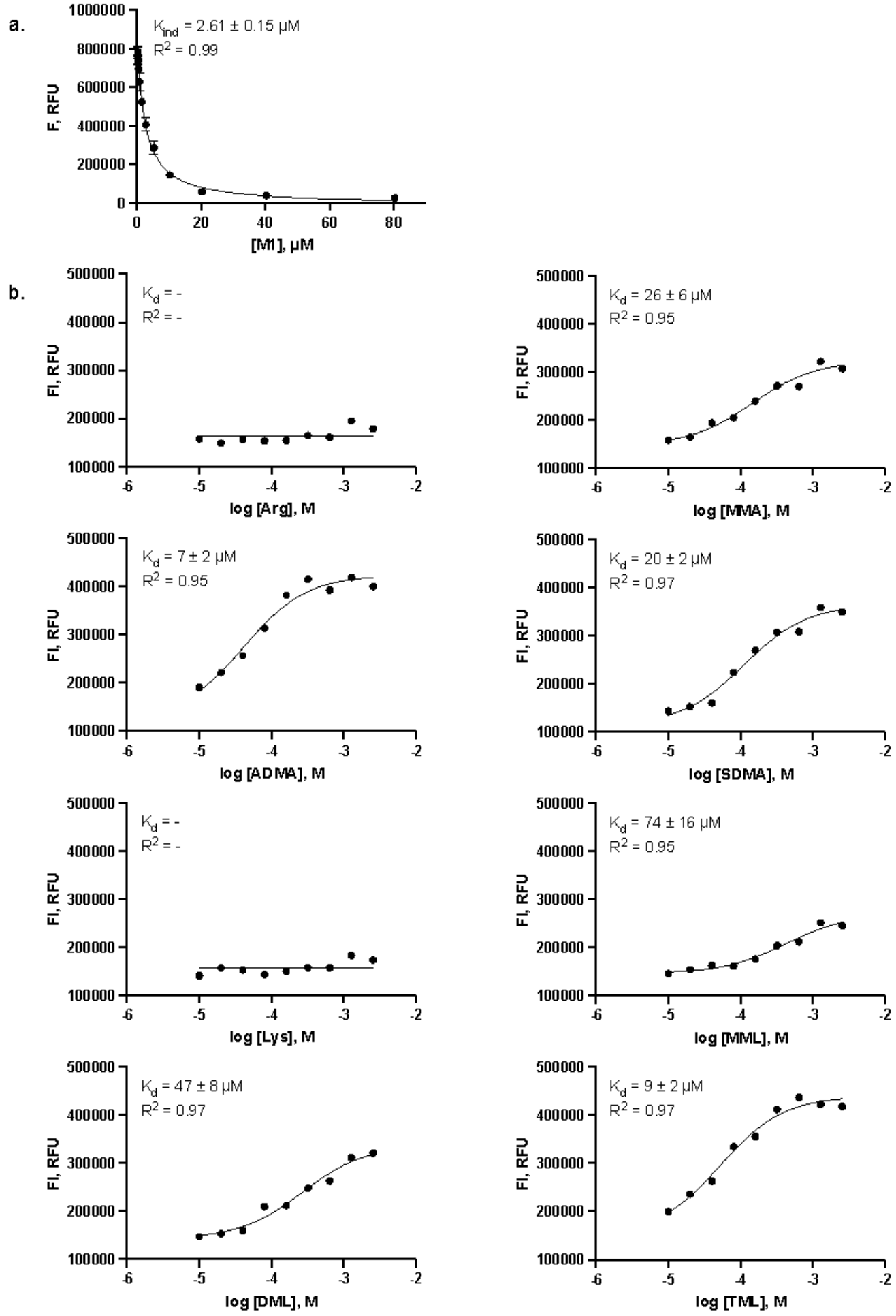

Figure S6: Fluorescence based studies of M1. a) Direct titration of R6G $(10 \mu \mathrm{M})$ with M1 $(0-80 \mu \mathrm{M})$. b) Competitive titrations between Arginine, MMA, ADMA, SDMA, Lysine, MML, DML and TML (0 - 2.5 $\mathrm{mM})$ individually titrated into the M1-R6G (10 $\mu \mathrm{M}$ M1, $10 \mu \mathrm{M}$ R6G) complex. 


\subsubsection{Fluorescence based studies of $\mathbf{M} 2$}

a.
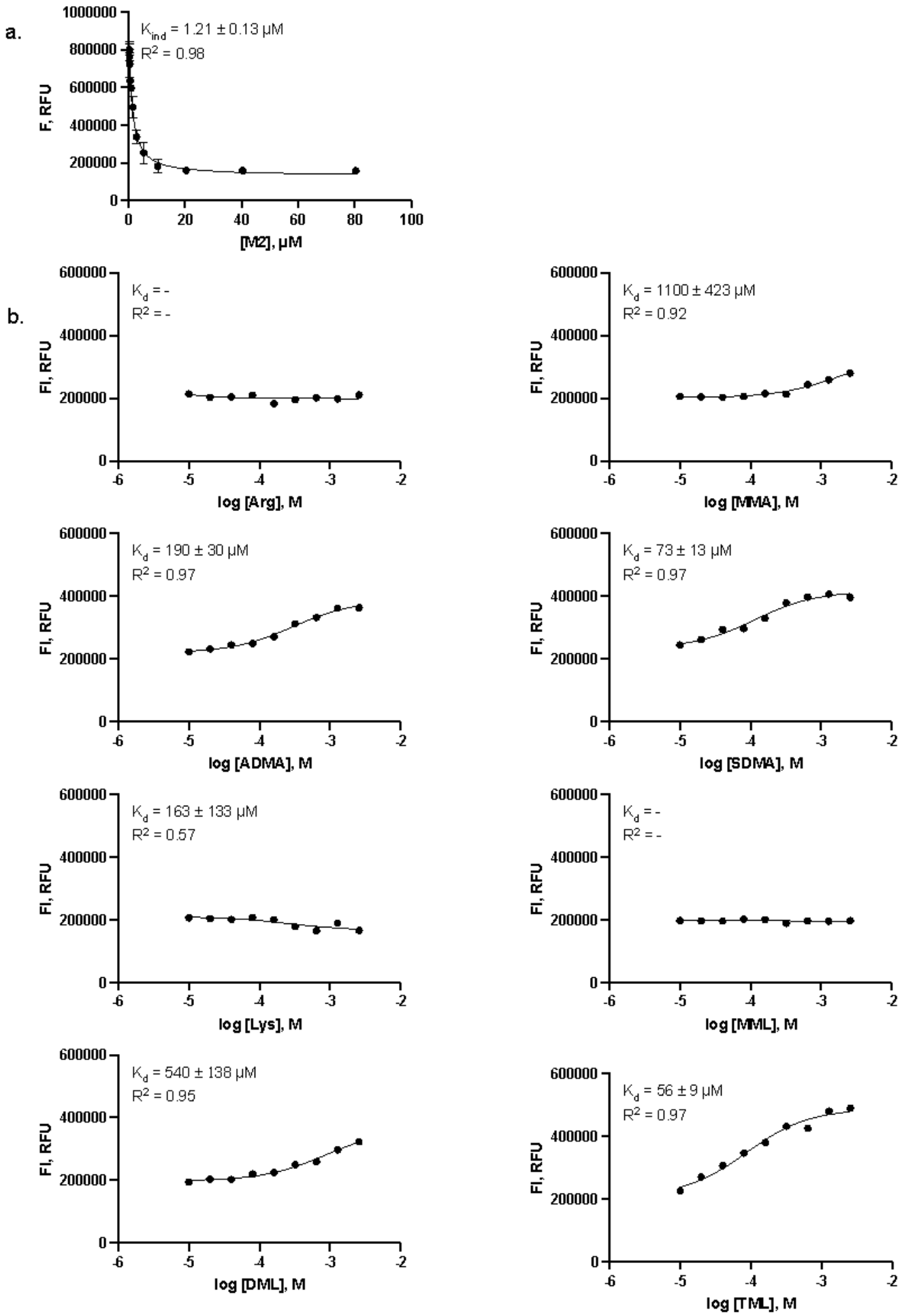

Figure S7: Fluorescence based studies of M2. a) Direct titration of R6G $(10 \mu \mathrm{M})$ with $\mathbf{M} 2(0-80 \mu \mathrm{M})$. b) Competitive titrations between Arginine, MMA, ADMA, SDMA, Lysine, MML, DML and TML (0 $2.5 \mathrm{mM})$ individually titrated into the M2-R6G $(10 \mu \mathrm{M}$ M2, $10 \mu \mathrm{M}$ R6G) complex. 


\subsection{Fluorescence based studies $50 \mathrm{mM}$ buffer}

\subsubsection{Fluorescence based studies of $\mathbf{M 1}$}
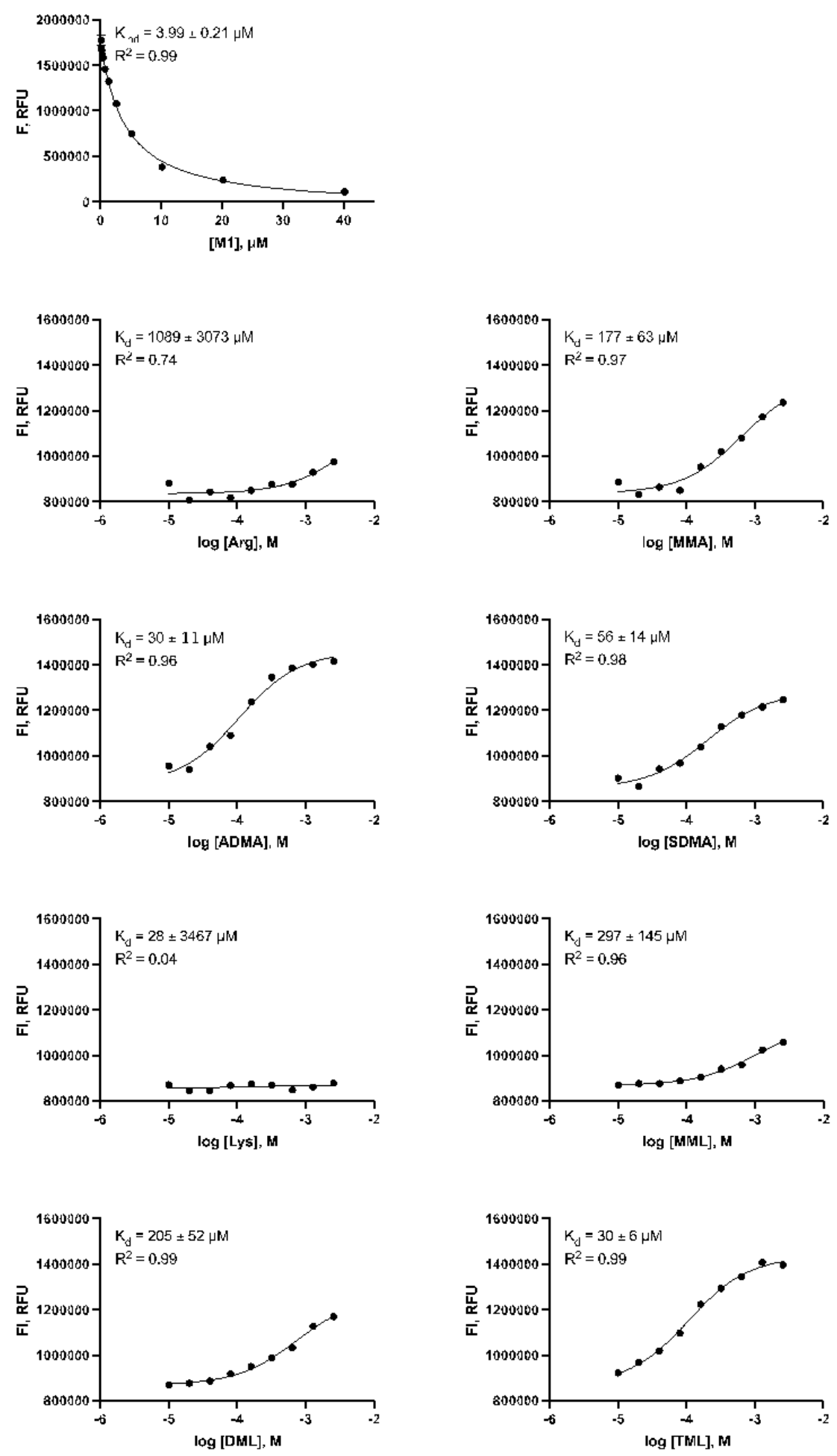

Figure S8: Fluorescence based studies of M1. a) Direct titration of R6G $(10 \mu \mathrm{M})$ with $\mathbf{M 1}(0-40 \mu \mathrm{M})$. b) Competitive titrations between Arginine, MMA, ADMA, SDMA, Lysine, MML, DML and TML $(0-2.5$ $\mathrm{mM})$ individually titrated into the M1-R6G (10 $\mu \mathrm{M}$ M1, $10 \mu \mathrm{M}$ R6G) complex. 


\subsubsection{Fluorescence based studies of $\mathbf{M 2}$}
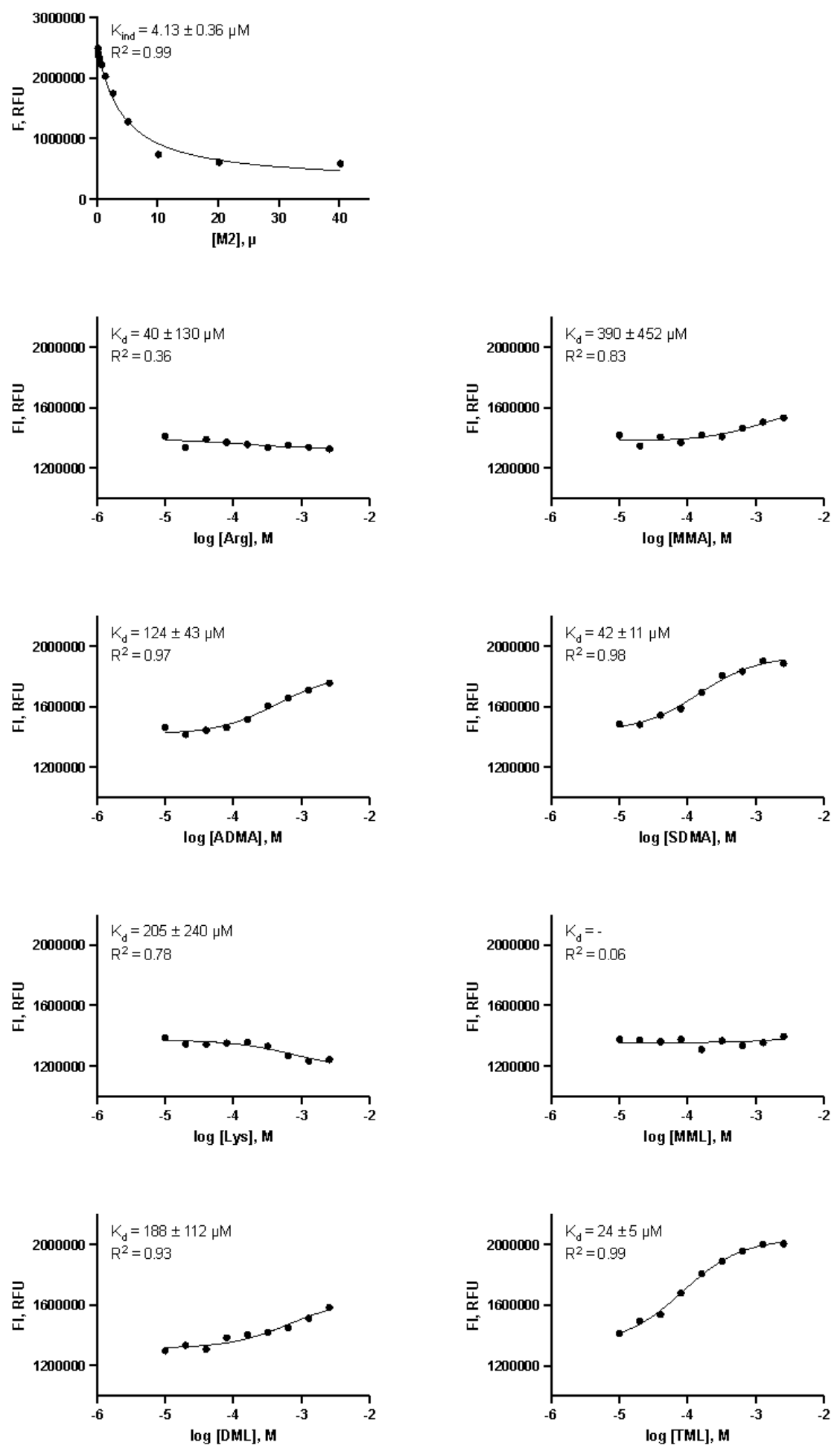

Figure S9: Fluorescence based studies of M2. a) Direct titration of R6G $(10 \mu \mathrm{M})$ with M2 $(0-40 \mu \mathrm{M})$. b) Competitive titrations between Arginine, MMA, ADMA, SDMA, Lysine, MML, DML and TML (0 - 2.5 $\mathrm{mM})$ individually titrated into the M2-R6G (10 $\mu \mathrm{M}$ M2, $10 \mu \mathrm{M}$ R6G) complex. 


\subsubsection{Fluorescence based studies of CLR01}
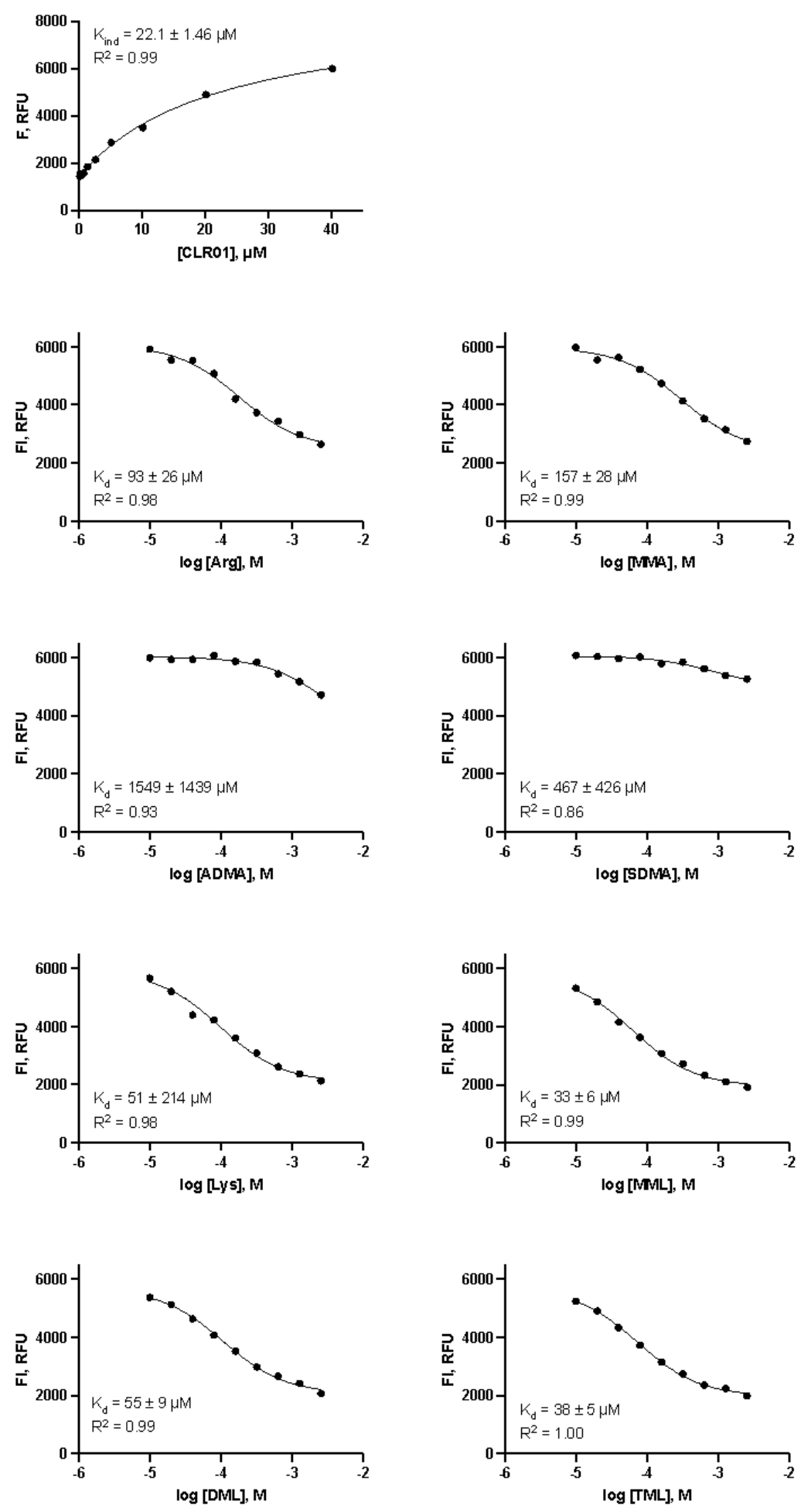

Figure S10: Fluorescence based studies of CLR01. a) Direct titration of 4-ASP $(20 \mu \mathrm{M})$ with CLR01 $(0-$ $40 \mu \mathrm{M})$. b) Competitive titrations between Arginine, MMA, ADMA, SDMA, Lysine, MML, DML and TML (0 - $2.5 \mathrm{mM})$ individually titrated into the CLR01-4-ASP (70 $\mu$ M CLR01, $20 \mu \mathrm{M} 4$-ASP) complex. 


\section{Molecular Modeling}

Modeling was done using minimization in explicit water for all the complexes (Force Field: OPLS_2005), in Maestro. Hosts are shown without hydrogen atoms and guests are shown with $\mathrm{H}$ atoms. Color Code for atoms: $\mathrm{C}=$ gray, $\mathrm{N}=$ blue, $\mathrm{H}=$ white, $\mathrm{O}=$ red, $\mathrm{P}=$ purple, $\mathrm{S}=$ yellow. Each host is modeled with ADMA (sCx4-CHO, sCx4-NO2, M1, M2, PC, CLR01). Hosts M1, M2, PC, CLR01 are also modeled with MML and with TML.

3.1. Each host modeled with ADMA. Front, back, top, and bottom views for all complexes.

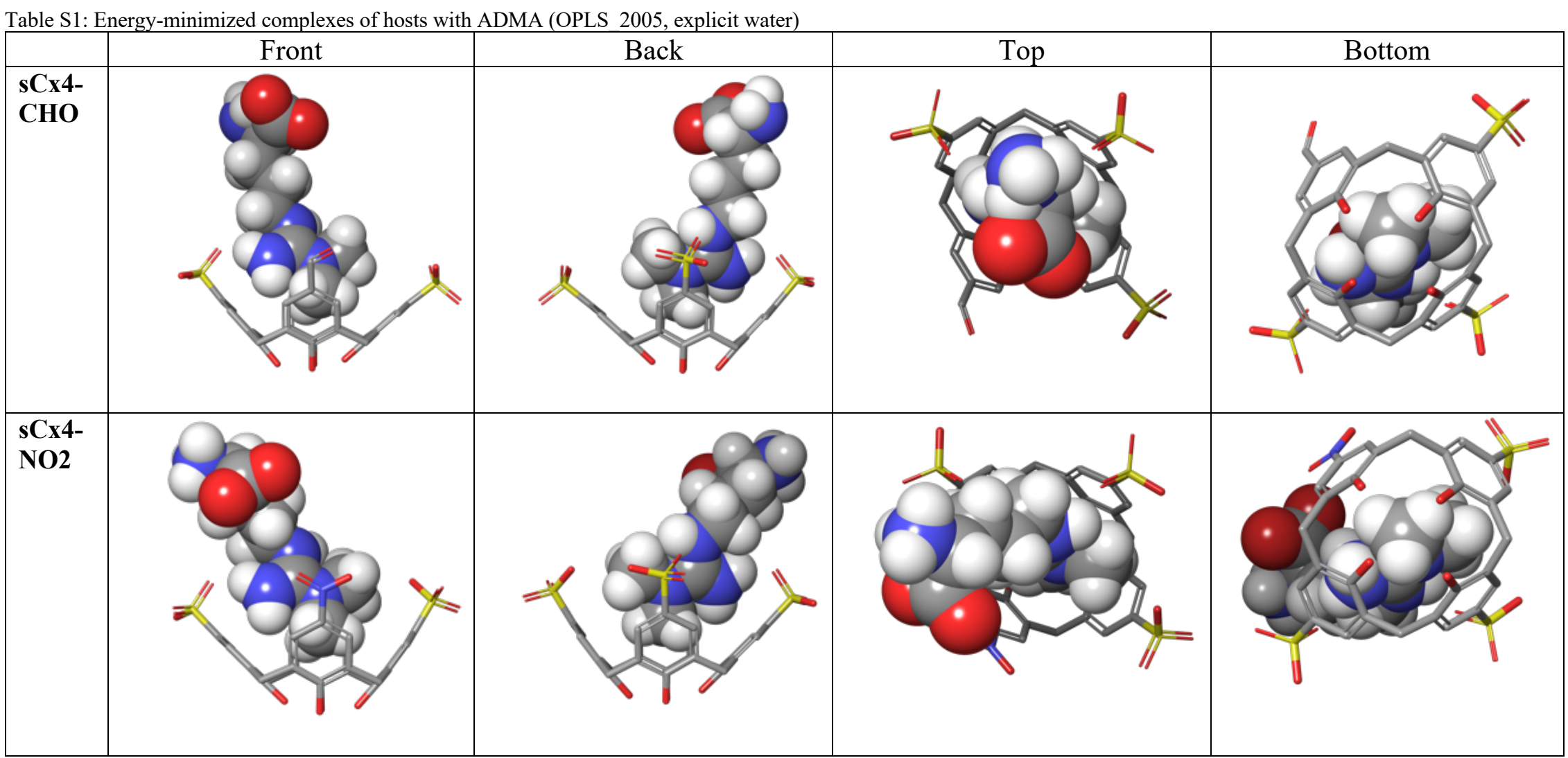




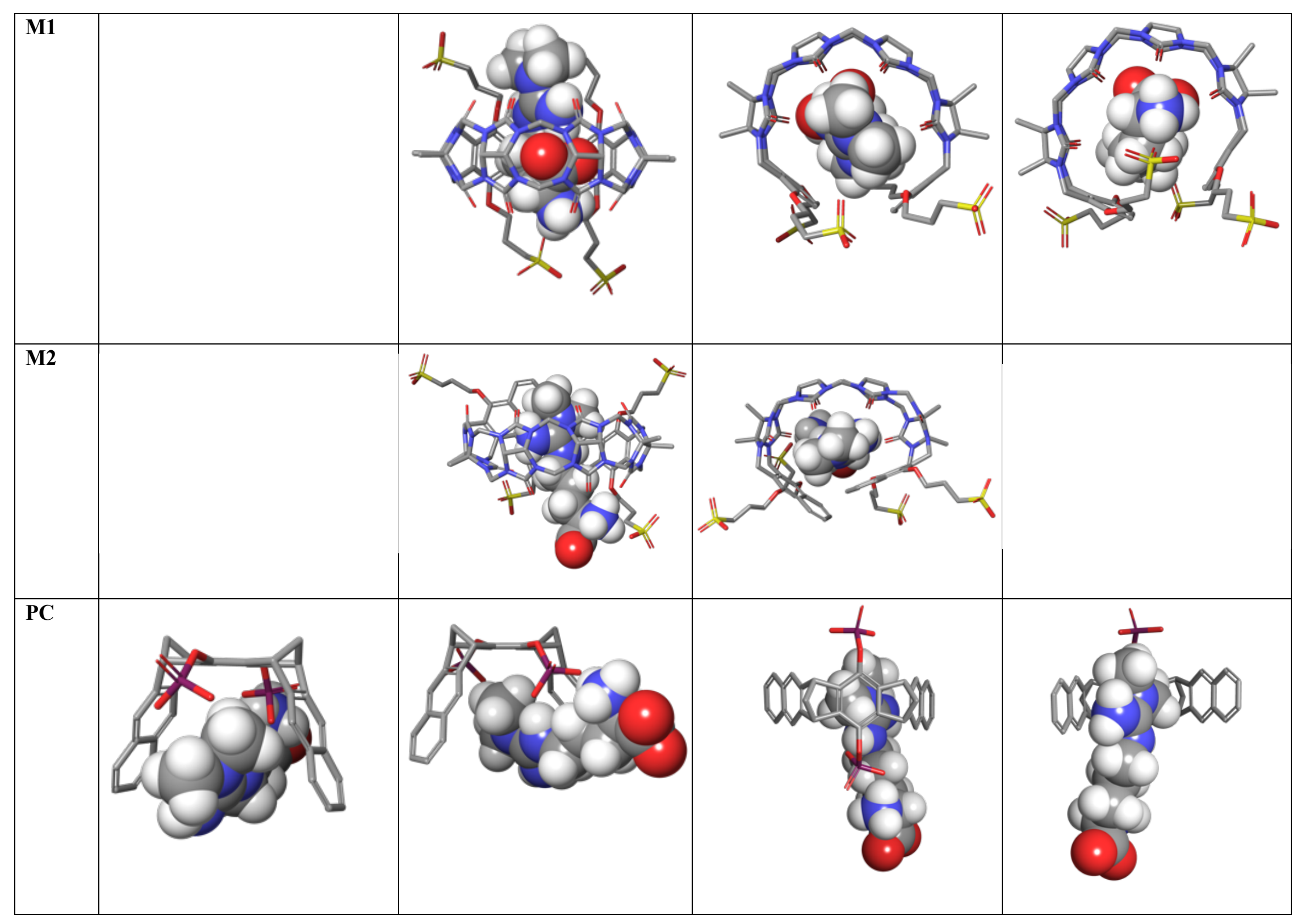




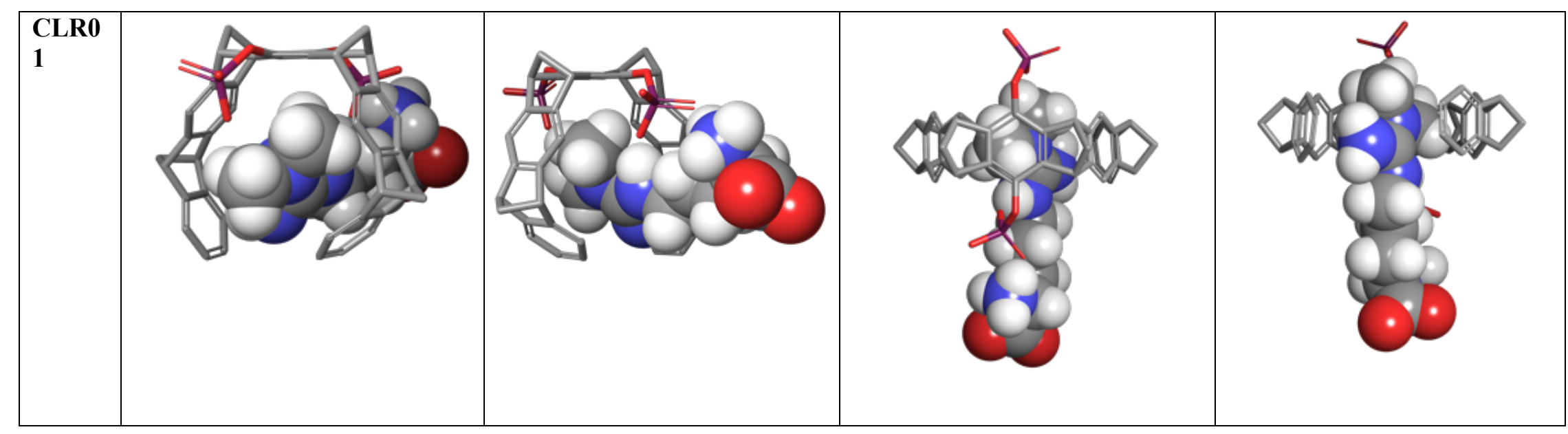


3.2. Hosts M1, M2, PC, CLR01 each modeled with MML and with TML.

Table S2: Energy-minimized complexes of host M1 with MML and TML (OPLS_2005, explicit water)


Table S3: Energy-minimized complexes of host M2 with MML and TML (OPLS_2005, explicit water)

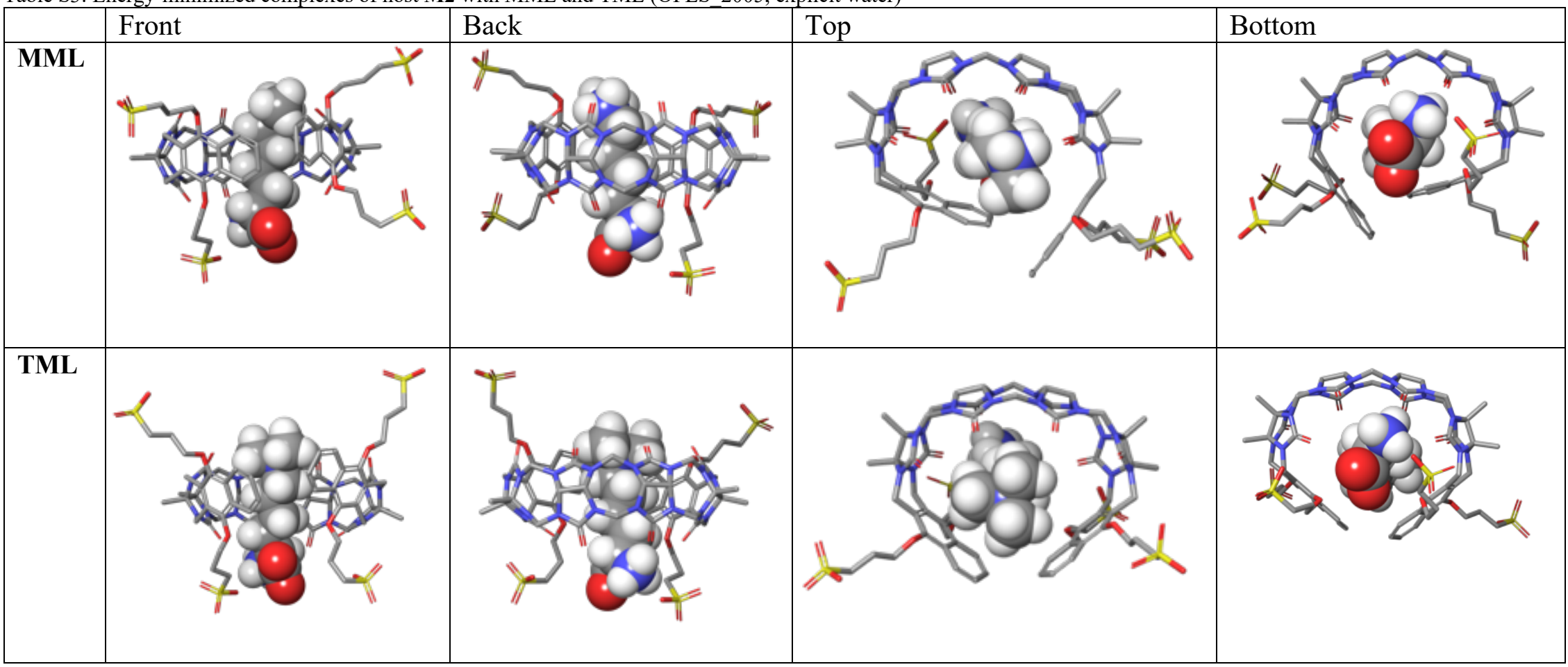


Table S4: Energy-minimized complexes of host PC with MML and TML (OPLS_2005, explicit water)

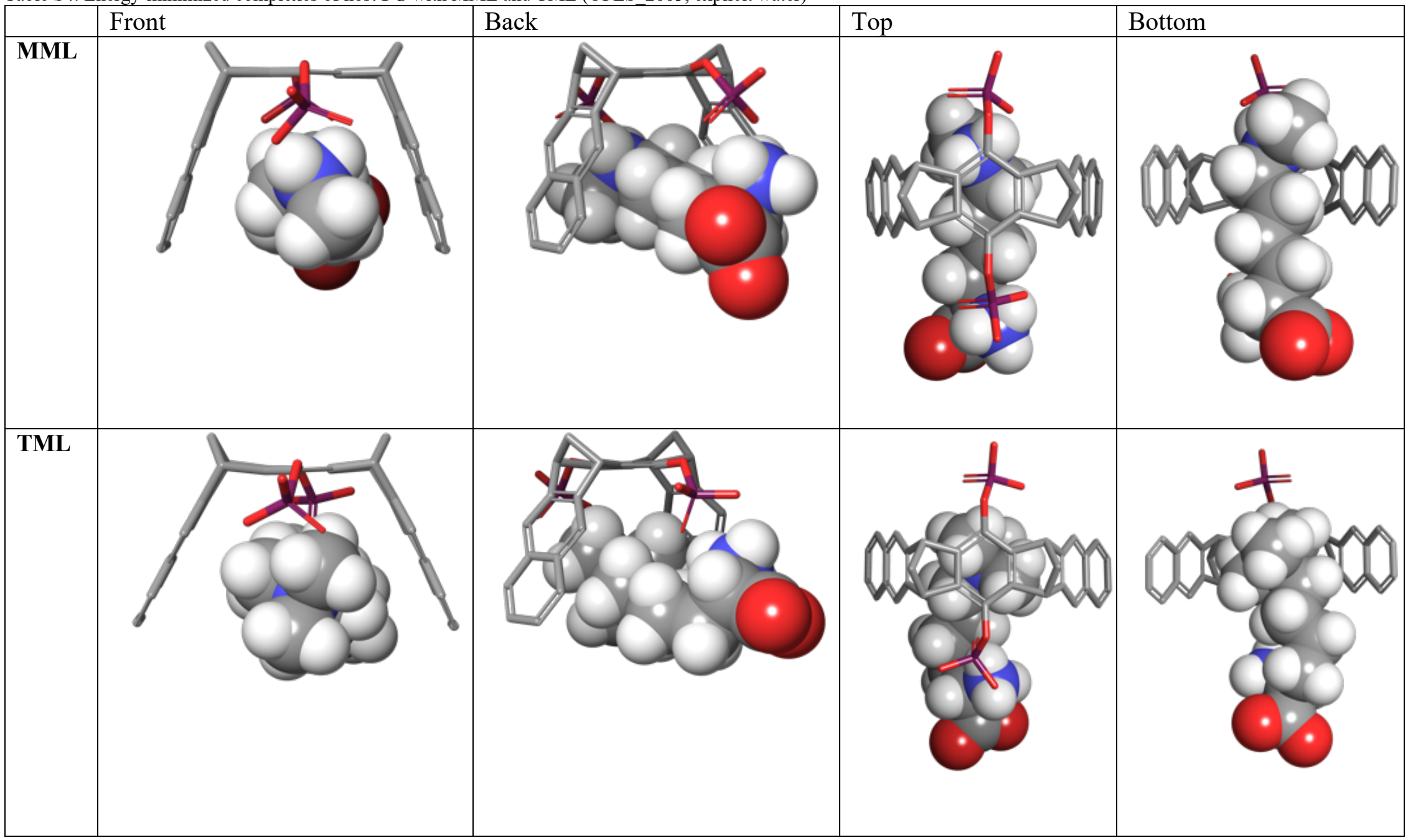




\begin{tabular}{l} 
Table S5: Energy-minimized complexes of host CLR01 with MML and TML (OPLS_2005, explicit water) \\
\hline MML
\end{tabular}




\section{References}

(1) Beatty, M. A.; Borges-Gonzalez, J.; Sinclair, N. J.; Pye, A. T.; Hof, F. AnalyteDriven Disassembly and Turn-On Fluorescent Sensing in Competitive Biological Media. J Am Chem Soc 2018, 140, 3500-3504.

(2) Daze, K. D.; Ma, M. C.; Pineux, F.; Hof, F. Synthesis of new trisulfonated calix[4]arenes functionalized at the upper rim, and their complexation with the trimethyllysine epigenetic mark. Org Lett 2012, 14, 1512-1515.

(3) Ma, D.; Hettiarachchi, G.; Nguyen, D.; Zhang, B.; Wittenberg, J. B.; Zavalij, P. Y.; Briken, V.; Isaacs, L. Acyclic cucurbit[n]uril molecular containers enhance the solubility and bioactivity of poorly soluble pharmaceuticals. Nat Chem 2012, 4, 503-510.

(4) Hadrovic, I.; Rebmann, P.; Klarner, F. G.; Bitan, G.; Schrader, T. Molecular Lysine Tweezers Counteract Aberrant Protein Aggregation. Front Chem 2019, 7, 657.

(5) Talbiersky, P.; Bastkowski, F.; Klarner, F. G.; Schrader, T. Molecular clip and tweezer introduce new mechanisms of enzyme inhibition. J Am Chem Soc 2008, 130, 9824-9828. 